Erschienen in: Stickel, Gerhard (Hrsg.): Deutsche Gegenwartssprache. Tendenzen und Perspektiven. - Berlin, New York: de Gruyter, 1990. S. 88-133. (Institut für deutsche Sprache. Jahrbuch 1989)

HARTWIG KALVERKÄMPER

\title{
Gemeinsprache und Fachsprachen - Plädoyer für eine integrierende Sichtweise
}

1. Kulturgeschichtliche Gegensatz-Relation:

Fachlichkeit und Laienschaft

1.1. Alltag als Lebensform

1.2. Fächer als Organisationsform

1.2.1. Formale Aspekte: Umfang und Anzahl

1.2.2. Inhaltliche Aspekte: Merkmale der Fachlichkeit

1.3. Fachlichkeit als Bezugsgröße für Laienschaft

1.3.1. 'Fach' unter sprach- und kulturgeschichtlichem Aspekt

1.3.2. 'Laie' unter sprach- und kulturgeschichtlichem Aspekt

1.4. Integration in einer Skala 'Fachlichkeits'-Merkmale

2. Sprachsystematische Gegensatz-Relation:

Fachsprachlichkeit und Gemeinsprache

2.1. Gemeinsprache und Fachsprachen

2.1.1. Fächer und (ihre) Fachsprachen

2.1.2. Laienwelt des Alltags und (ihre) Gemeinsprache

2.1.2.1. Modellvorstellung: sektoral und relational

2.1.2.2. Merkmale der Gemeinsprache

2.1.2.3. Verständlichkeit und funktionale Modellvorstellung

2.2. Beziehung von Gemeinsprache und Fachsprachen in referentiellen Texten

2.2.1. Konsequenzen gegen die Kategorie 'Gemeinsprache'

2.2.2. Kritik an der Kategorie 'Gemeinsprache' in der Fachsprachen-Forschung

2.2.2.1. Sicht auf Sprachsystem: „Gesamtsprache”

2.2.2.2. Sicht auf Textsorten und Sprachverwendungssituationen: Kommunikative Reichweite von Fachsprache

2.2.2.3. Sicht auf Varietäten: „Alltagssprache”

3. Integration in einer Skala der 'Fachlichkeits'-Merkmale

3.1. Chancen und Perspektiven

3.1.1. Traditionelle Methode: Sammeln und Zuweisen

3.1.2. Ausweg: Skala der 'Fachlichkeits'-Merkmale

3.1.3. 'Fachsprachlichkeit' als Eigenschaft der Skala

3.1.3.1. 'Fachsprachlichkeit' als Orientierung

3.1.3.2. Die gleitende Skala

3.2. Instrumente und Methode

3.2.1. Merkmale der Fachsprachlichkeit: Vom Wort zum Text-in-Funttion

3.2.2. Methodischer Gewinn 


\section{Kulturgeschichtliche Gegensatz-Relation: \\ Fachlichkeit und Laienschaft}

\subsection{Alltag als Lebensform}

Es gibt, so sagt eine bekannte Weisheit, wohl nichts Schwierigeres, als etwas anscheinend Einfaches und Alltägliches zu beschreiben und zu definieren. In der Tat bietet die Lebenspraxis immer wieder Gelegenheiten, die Gültigkeit dieser Beobachtung zu erfahren: Wie schmeckt ein Pfirsich, warum gibt die Kuh Milch, warum dampft kochendes Wasser, wieviele Haare hat ein Mensch, was ist 'Liebe', was ist 'schummrig', wieso wächst man - solche Fragen, meist aus Kindermund, sind eine Herausforderung an die Erklärungsfähigkeiten der Eltern; auch die Wissenschaften kennen da ihre Schwierigkeiten, wenn sie auf vermeintliche Selbstverständlichkeiten ihrer Objekte hin angesprochen werden: Was heiBt 'Elemente', 'Teilchen', was besagt 'Kranksein', was ist ein 'Wort', ein 'Satz', wieviele Sprachen gibt es, und wieviele Wörter hat eine Sprache?

Wenn aber das Alltägliche, das Selbstverständliche, nun selbst als solches befragt wird, erschwert sich das Antworten noch um ein Vielfaches. Auch hier bestätigt sich die bekannte Einsicht von Bertrand Russell, 1948 in „Human knowledge, its scope and limits" (London) ausgesprochen, da 8 „die Sprecher beim normalen Sprechen nicht mehr Grund [haben], sich der Wortbedeutungen (und überhaupt der Strukturen ihrer Sprache) bewußt zu werden, als die Planeten Anlab haben, sich auf ihren Umläufen der Keplerschen Gesetze zu erinnern" (Hard 1970, S. 36). In der Tat ist es ein noch ungewohnter Aspekt, den Alltag als einen interessanten Bereich wahrzunehmen, den es als solchen zu hinterfragen lohnen könnte. Alltag lebt man, aber man beschäftigt sich nicht oder nur kaum mit ihm, geschweige dab man sich um ihn oder gar über ihn Gedanken machen würde.

Die breite Einstellung dem Alltag und seinen Alltäglichkeiten gegenüber ist - fafbar seit der Zeit des Sturm und Drangs - deutlich negativ besetzt: Man spricht vom "grauen Alltag", vom ngewöhnlichen Einerlei des Lebens" und meint das ereignislose Dasein, die Trivialität der Situationen und die Banalität des Sprechens, die Üblichkeiten des Handelns, eben nichts Besonderes im Lebensablauf.

Aus der Gegenwelt des Alltags heraus, nämlich aus der Welt der spezifischen Anforderungen, des differenzierten Handelns, der interessanten Abläufe, haben Ende der sechziger, verstärkt in den siebziger Jahren die Soziologen den Lebensbereich der interesselosen Routine, der unspezifischen Situationen und die Lebensform der "Verödung und Entfremdung" (Weinrich 1985 [1981] , S. 266) entdeckt. Der Alltag wurde zu einer Her- 
ausforderung für den sezierenden Geist, für den klassifizierenden Wissenschaftler: Die Alltagsforschung entstand, als eine ${ }_{n}$ Phänomenologie der Lebenswelt" von Alfred Schütz zu einer empirisch-soziologischen Methode entwickelt und von Harald Garfinkel, Aaron Victor Cicourel und anderen zu einer "Ethnomethodologie", einer "Soziologie des Alltagshandelns", 1 ausgebaut.

Auch die Juristen waren gezwungen, sich mit dem Phänomen des Alltäglichen auseinanderzusetzen, da sie den Begriff des Alltags als eine Bezugsgröße bei der Rechtsfindung benötigten: So orientiert sich das Warenzeichenrecht an Kategorien wie 'Allgemeine Meinung', 'Durchschnittskäufer' oder 'herrschende Verkehrsauffassung' zur Abwägung von Interessenkollisionen im Wettbewerb; so nimmt der juristische Kommentar sein $\mathrm{MaB}$ an dem ,vernünftigen und redlichen Durchschnittskunden”, wenn über die Verständlichkeit von Vertragsbedingungen befunden werden soll. ${ }^{2}$

Es sind gerade die pragmatischen Anforderungen, die den Blick auf den sonst kaum wahrgenommenen Alltag zwingen und es somit erreichen, daB sich für eine verschwommene Kategorie Konturen ausprägen und dab ein als undifferenziert empfundenes Dasein und routinehaftes, unspezifisches Handeln ein neues Profil bekommt:

So hatten im besonderen die Juristen aus der Branche der Haftpflichtversicherungen lebhaftes Interesse daran, die täglichen Anforderungen an eine Mutter, ihre üblichen Tätigkeiten und alltäglich erbrachten Leistungen im Haushalt kennenzulernen. ${ }^{3}$ Denn vor der traurigen Bilanz, daß jährlich 8.000 Hausfrauen und Mütter bei Verkehrsunfällen verletzt und 800 getötet werden, galt es, für den jeweiligen Zuspruch von angemessenem Schadensersatz jenes allbekannte und gerade deshalb so unbekannte Betätigungsfeld 'Haushalt' auszuloten. Die Ergebnisse der Untersuchungen korrigieren zwingend die verbreitete Meinung, für Haushalt bedürfe es keines besonders ausgewiesenen Wissens, keiner speziellen Kenntnisse. Vielmehr umfaBt das "Anforderungsprofil”, wie es die Arbeitswissenschaftler erstellt haben, ein breites Spektrum differenzierter und qualifizierter Tätigkeiten, Entscheidungen, Planungs- und Organisationsarbei-

1 Arbeitsgruppe Bielefelder Soziologen 1973, Cicourel 1964, Garfinkel 1967, Goffman 1974, Hammerich/Klein 1978, Laermann 1975, Weingarten/Sack Schenkein 1976, Weinrich 1985 [1981], S. 275 f., Wierlacher 1980.

2 Kalverkämper 1983, S. 138.

${ }^{3}$ Aktueller Bericht von ADAC motorwelt 1 (Januar), 1989, S. 48-51: „Wieviel ist eine Mutter wert?" 
Was allgemein und üblicherweise als Prototyp des unspezifischen Handelns, der normalen Tätigkeiten, des interesselos grauen Einerleis, der Routine ohne notwendige Kennerschaft gilt und entsprechend auch in Befragungen eingeschätzt wird, ${ }^{5}$ entpuppt sich, empirisch verfolgt, als eine dichte Überschneidung und Abfolge von - gerade gegenteilig - speziellen Arbeiten in den verschiedensten Bereichen und mit unterschiedlichen Zielsetzungen. Immerhin repräsentiert wohl jede dieser im Haushaltsalltag wechselnden Tätigkeiten, außerhalb der Grenzen von Wohnung und Haus, einen eigenständigen Beruf, eine spezialisierte Arbeit, eine fachlich ausgebildete Betätigung: Das Kochen macht professionell der Koch, das Backen hat als Beruf der Bäcker erlernt, das Putzen und Waschen führt sachgerecht der Reinigungsfachmann aus, die Erziehung ist beruflich Sache des Pädagogen, Gartenarbeit ist Teil der Tätigkeiten im Gärtnerberuf, usw. Allerdings die Arbeiten, die im Schnittpunkt solcher fachlicher Tätigkeiten liegen, nämlich die Hausarbeit, dann auch konsequenterweise als 'Beruf', als fachliche Tätigkeit einzustufen und entsprechend zu bezeichnen, fallt bis heute schwer.

Diese Einschätzung teilen die Hausarbeit und die Tätigkeiten im Umfeld universaler Lebensbedürfnisse mit Bereichen wie Hobby und Freizeit, Mode, Kunst, Umweltschutz, verschiedenen Sportarten (zum Beispiel Schwimmen oder Radfahren), Reisen, Essen und Trinken, Sexualität und wenigen anderen. ${ }^{\circ}$ Allerdings: Auch hier werden Handlungen durchgeführt, zielgerichtet, komplex, mit speziellen, oft sehr intensiv erworbenen Kenntnissen, für die es, gleichsam als manifesten Beweis, Sachbücher und Lernmedien, Ausbildungskurse und Fortbildungseinrichtungen gibt.

1 Aufgezählt werden Kleinkinder, Hausaufgaben, Freizeit, Pflege, Kontakte, Führung, Hausreinigung, Geräte, Kleingeräte, Spülen, Wäschereinigung, Anfertigung, Einkauf Lebensmittel, sonstige Einkäufe, Gartenarbeit. Es fehlen meines Erachtens noch: Umgang mit Behörden und Ämtern, Verkehr mit Institutionen wie Bank, Schule, Freizeiteinrichtungen für die Kinder, sowie Konsultationen von Ärzten für das Kind.

5 Im Rahmen einer gezielten Befragungs- und Fragebogenaktion zum 'Fach'Begriff in verschiedenen Universitätsstädten (s. Anmerkung 7) habe ich auch zum Begriff des 'Alltags' Fragen gestellt, deren Beantwortung in die Darstellung hier einbezogen werden. Siehe auch Weinrich 1985 [1981], S. 266, 286 Anm. 6.

6 Aufzählung aus dem Ergebniskorpus der Fragebogen-Aktion. Ich beabsichtige, die Auswertung der Befragung zum 'Alltags'-Begriff in späterer Zeit zu veröffentlichen, und zwar in Verbindung mit den Resultaten der Fragebogenund Befragungs-Aktion zu den Begriffen 'Fach' und 'fachlich' bzw. 'Fachlichkeit'. 
Aber die öffentliche Meinung schätzt sie nicht als Tätigkeiten ein, denen eine berufliche oder fachliche Qualität zugesprochen werden könnte, vielmehr werden sie prinzipiell als allgemein, allbekannt, alltäglich, als unspezifisch eingestuft; daß diese Urteile ungerechtfertigt sind, legt die Ethnomethodologie nahe (zum Beispiel Goffman 1977), indem sie bei Alltagssituationen die „besonders hohe Komplexität des Zusammenwirkens historischer, ökonomischer und kultureller Faktoren" (Picht 1989, S. 57) hervorhebt. „Bei näherer Betrachtung ist Alltag also keineswegs alltäglich" (loc. cit.).

\subsection{Fächer als Organisationsform}

Während die Alltagskultur offenkundig nicht zum bewußten Alltagswissen selbst gehört, ist auf der anderen Seite die Öffentlichkeit rasch und recht mühelos imstande, die Frage nach Fächern und Berufen zu beantworten:

\subsubsection{Formale Aspekte: Umfang und Anzahl}

An der Spitze einer längeren Liste ${ }^{7}$ liegen - den Ersterfahrungen entsprechend - Schulfächer (Mathematik, Deutsch, Sprachen, Biologie, Physik, Sport, Chemie), danach Philosophie, Psychologie, Pädagogik, Soziologie, Politikwissenschaften, Linguistik, Germanistik, Romanistik, Anglistik, Literaturwissenschaften und der medizinisch-naturwissenschaftliche Bereich.

Eine solche Aufzählung, von den Befragten auf weite Strecken übereinstimmend gewählt, erhellt natürlich auch den Konsens, die Sicherheit und die Selbstverständlichkeit, mit denen die Qualität, ein 'Fach', eine fachliche Tätigkeit zu sein, im öffentlichen Bewußtsein verankert ist. Vorweg haben die sogenannten Prestige-Berufe, die mit einem besonderen Nimbus und mit einer hohen Erwartungshaltung umgeben sind - oder sich umgeben haben? (eine Sozialgeschichte der Prestige-Berufe wäre noch zu schreiben) -, die Einschätzungen mitgeprägt, die insgesamt den Begriff des 'Faches' bzw. der 'Fachlichkeit' von Objekten, Sachverhalten und Handlungszusammenhängen ausmachen. ${ }^{8}$

Welche Fächer nun auf jeden Fall aufzuzählen seien, läBt sich verbindlich

7 Fragebogen-Aktionen in Freiburg, München, Köln und Hagen mit studentischen Probanden und mit Zufallspublikum (FuBgängerzone).

8 Zu diesem soziokulturell, europäisch-begriflich und einzelsprachlich sehr interessanten Fragenkomplex habe ich gezielt Forschungen betrieben: Kalverkämper $1980 \mathrm{~b} ; 1989 \mathrm{a} ; 1990$. 
nicht beantworten; nach welchem Maßstab sollte man auch die intuitiv sprudelnden Auflistungen der Befragten steuern oder gegebenenfalls korrigieren? Nach der Frequenz? - aber welche Gelegenheiten und welche Erscheinungsformen für das Auftreten des Fachlichen wären dann dazu zu wählen? Nach der Relevanz? - aber wie bemißt sie sich, und in bezug auf wen soll der Relevanzgesichtspunkt gelten? Schlieflich: nach der Tradition?, und das heift auch: nach aktuell geltender Meinung? Doch die streitet sich noch über die Anzahl von Fächern.

Vielleicht wären die erstaunlichen Übereinstimmungen in den allgemeinen einsprachigen Wörterbüchern eine Leitvorgabe: denn im Vergleich der fachlichen Markierungen ihrer Wortschätze stellt sich heraus, dab es offenbar einen Fächerkanon der Lexikographie gibt, mit 22 durchweg angesetzten Fächern oder Sachbereichen:早 Architektur, Anatomie, Astronomie, Botanik, Biologie, Chemie, Geologie, Geographie, Grammatik, Mathematik, Medizin, Militär, Mineralogie, Musik, Philosophie, Physik, Physiologie, Psychologie, Religion/Theologie/Kirche, Sprachwissenschaft, Technik/Technologie, Zoologie. - Oder man stützt sich auf Klassifizierungsversuche, die sich statistisch an Textkorpora ausrichten; oder die sich an der Menge der Buchtitel-Produktion orientieren; oder die eine spezifische theoretische Evidenz beanspruchen; ${ }^{10}$ oder die praktischorganisatorisch (zum Beispiel im Buchhandel) erprobt sind. ${ }^{11}$

Man kann natürlich auch der von Eugen Wüster, dem Nestor der Terminologielehre, begründeten Tradition folgen und seine Schätzung übernehmen, die auf ungefähr 300 Fachbereiche (wie Betriebswirtschaft, Volkswirtschaft, Anatomie, Pharmazie u.a.) lautet. ${ }^{12}$ Praxisbezogene Aktivitäten haben ihre eigenen Fächerkanones tradiert, so die datenverarbeitenden und dokumentationsintensiven Arbeiten in der Wissenschaft (Wissensdarstellung), in der Wirtschaft (Wissensverwendung und Wissensvermittlung) und in der Verwaltung (Wissensorganisation), bei de-

- Kalverkämper 1989b. Diese Ergebnisse sind unmittelbar aus den Entscheidungen von deutschen, französischen, englischen und italienischen einsprachigen allgemeinen Wörterbüchern herausgefiltert, welche ,diatechnischen Markierungen" (Angaben zur Fach[sprachen-] zugehörigkeit) sie wählen und wie sie sie praktisch einsetzen.

10 Cf. Harald Weinrichs Vorschlag für ein neuartiges interdisziplinäres Wörterbuch (Weinrich 1976b; 1978).

$11 \mathrm{Zu}$ diesen Versuchen s. Mentrup 1978b, spez. S. 213-221 mit ausführlichen Auflistungen und instruktiven Tabellen aus der einschlägigen Forschung.

12 Wüster 1973; s. aktuell auch Felber/Budin 1989. 
nen eine Fächer-Anzahl von 857 bis 3.000 Fächern errechnet ${ }^{13}$ und Fächerkataloge mit etwa 2.500 bis zu 7.000 Fächern verbindlich sind. ${ }^{14}$

\subsubsection{Inhaltliche Aspekte: Merkmale der Fachlichkeit}

So scheint es bislang nur möglich zu sein, Abgrenzungen von Fächern und Berufen zueinander - und somit ihre Anzahl und ihren jeweiligen Umfang - nur im einzelnen Bedarfsfall vorzunehmen und dabei Kriterien wie 'soziale Konvention', 'pragmatische Zwänge', 'sachliche Evidenzen', 'Normungsdruck', 'Komplexität des Objektbereichs', 'innerer Zusammenhalt von Handlungsabläufen', 'Lehr- und Ausbildungstraditionen' und mögliche weitere einzusetzen. Inhaltlich finden derartig geleitete Einstufungen als 'Fach' oder 'fachlich' breiten Konsens; das liegt darin begründet, daf es offenbar konstante Merkmale der Fachlichkeit gibt, die sowohl dem befragten Durchschnittsmenschen auf der Straße, als auch dem professionell mit und in Fächern Beschäftigten bewußt sind.

\section{So gelten als Merkmale des Fachlichen}

- die besondere Kenntnis in der (erwerbsmäßigen) Arbeit, wie man sie sich in Ausbildung, Lehre, Studium aneignet und dabei spezifische Handlungsabläufe, Umgangs- und Kommunikationsgewohnheiten, Arbeitsverteilungen und Zuständigkeiten kennenlernt; dies entwickelt ein Bewußtsein für Methoden und Arbeitsprozesse, für funktionierende Zusammenhänge der Tätigkeiten auf ein bestimmtes Produktionsziel hin;

- des weiteren das spezialisierte Wissen zu einem Sachgebiet und Handlungsraum; es verschafft tiefere Einsicht in sachliche Zusammenhänge und ermöglicht es, einzelne Gegebenheiten systematisch zu erfassen und einzuordnen.

Mit diesen Merkmalen - 'Fähigkeit', 'erworbene Kenntnis', 'Fertigkeit' und 'Wissen' - werden Tätigkeiten abgewogen und mit ihren Handlungsräumen als 'Fächer' eingestuft, werden lebenspraktische Arbeiten als 'Berufe' konventionalisiert, werden aber auch andererseits - daran gemessen und dazu gleichsam negativ definiert - Betätigungen wie Hausfrauenarbeit, wie das Sexualleben, wie Essen und Trinken, wie das Reisen als nicht-fachlich, als allgemein und alltäglich befunden.

13 Siehe Mentrup 1978a, spez. S. 224.

14 Cf. zum Beispiel Dahlberg 1974 oder Mentrup 1978b. Ich erspare mir hier Einzelaufzählungen und verweise bündelnd auf Grundlagenwerke wie Hoffmann 1985 oder Fluck 1985. 


\subsection{Fachlichkeit als Bezugsgröße für Laienschaft}

Ein solches Einteilen von Handlungen in zwei Kategorien - fachliches Handeln einerseits und nicht-fachliches Handeln andererseits - entsteht nicht aus Zufällen. Kategorien sind, das wissen wir spätestens seit Aristoteles, relationale Ordnungsgrößen; sie werden eingesetzt 'in bezug auf' prós $t i^{15}-$, ganz dem griechischen Etymon entsprechend, nach dem die kategoria 'Anklagepunkt', 'Beschuldigung' bedeutet, also von jemandem auf jemanden hin - auf den Kontrahenten nämlich - zielt. Auch dieser gegnerische, der Gegensatz-Aspekt liegt hierin beschlossen und verweist auf die moderne Erkenntnis der Neurophysiologen und der Psycholinguistik, daB die Basiskategorie unseres klassifizierenden Denkens (Assoziieren im Wortfeld) und Sprechens (Wortwahl in Sätzen und Texten) die semantische Opposition, die Antonymierelation, ist. ${ }^{16}$

Von daher scheint es somit durchaus plausibel, daß sich durch die Menschheitsgeschichte hindurch die kategorische Trennung in fachliches und nicht-fachliches Handeln festgesetzt hat und als solche unangetastet blieb.

\subsection{1. 'Fach' unter sprach- und kulturgeschichtlichem Aspekt}

Die ältere von beiden ist mit Sicherheit die Kategorie der Fachlichkeit. Thre Ursprünge gründen in den Organisationsformen der Urgemeinschaften: Hier hatten das Teilen, der Anteil, das Zumessen von Jagdbeute und des Ertrages der Arbeit eine zentrale Funktion inne und prägten die Vorstellung vom genossenschaftlichen Ring aus, von der gegenseitig kreisenden Arbeitshilfe, vom Zaun aus Menschen, der nach außen hin schützt und nach innen hin bewahrt, vom hegenden Mannring. Der Altgermanist Jost Trier hat diese kultursoziologischen Verhältnisse aus Ringwörtern, wie ping, conventus, communitas und vielen anderen herauspräpariert ${ }^{17}$ und als dahinter verborgene Grundanschauung den Zaun erkannt, mystisch als gemeinschaftsbildender Menschenzaun, als Mannring, und in konkreter handwerklicher Umsetzung als Schutz aus eingerammten Balken, Verflechtungen und Lehmausschmierungen. Ganze Wortsippen haben von hier ihren Ausgang genommen, wie 'Seite' der geflochtenen Hauswand, aus der Wurzel 'sej-; wie 'Lehm', 'Leim', 'limes' und andere aus der Wurzel "lein-; wie 'Werk', 'wirken',

15 Ich denke speziell an die "Kategoriai" des Aristoteles, dort Stellen wie 5b, $16 \mathrm{ff} ., 20,29$ und weitere.

16 Hierzu zum Beispiel Raible 1981.

17 Trier 1951; 1981: speziell Aufsatz 10, 11, 12, 14. 
franz. guerche und andere aus der sprachlichen Zaun- und Flechtwurzel "uer'̧-; wie 'ritzen', 'reißen', engl. write, german. writan und andere aus der reichen Wurzel *uer-. In diese Gemeinschaft gehört auch die indogermanische Wurzel ${ }^{*} p \vec{a} \hat{g}-$, ${ }^{*} p \vec{a} \hat{k}$-, aus der sich über griech. pëgnýnai 'festmachen', 'zusammenfügen', 'befestigen' und über gleichbedeutend lat. pangere und pagus 'Gau', 'Gemeinde' die romanischen Wörter für den Begriff 'Land' entwickelt haben: so franz. pays, span./port. pais, ital. paese. Ihnen liegt der arbeitsweltliche Gehalt des hegenden Rings, die handwerkliche Vorstellung vom Einrammen, verflechtenden Zusammenfügen und von schützender Ausgrenzung noch zugrunde. Die Weiterentwicklung in den germanischen Sprachen - so altsächs. fac, angelsächs. foec, althochdt. fah - verweist ebenfalls auf die ursprünglichen handwerklichen Tätigkeiten: auf 'Abteilungen', 'Abgrenzungen', eben neuhochdeutsch auf 'Fächer', wie sie die Bauhandwerker, Fischer und Bauern für ihre beruflich-fachlichen Zwecke wie Fachwerkbau, Wehrbau, Fangkästen, Speicherkisten usw. einsetzten.

Mit den Fächern ging die Arbeitsteilung als eine kulturausprägende Kraft der historischen Gesellschaften einher, und Bauern, Fischer, Waffenhandwerker, Heilkundler, Jagdkundige, Bauhandwerker, zur See die Seefahrer, dann auch die Kundigen des Bergbaus und der Verhüttung von Erzen schafften die handwerklich-praktische Grundlage dafür, daß sich eine Kategorie des fachlichen Betätigens kulturgeschichtlich eigenständig hat herausbilden können. Es sei nicht vergessen, daß - im europäischen Kulturkreis - sich bereits in der Renaissance, dann aber systematisch und disziplinenbewuBt im Aufklärungszeitalter, auch eine wissenschaftlichtheoretische Fachlichkeit entwickelt und der handwerklich-praktischen zugesellt hat.

\subsection{2. 'Laie' unter sprach- und kulturgeschichtlichem Aspekt}

Einer Kategorie, die die Tätigen in ihrer Arbeit umgrenzt, mit „Zäunen” abschirmt und als Gruppe der fachlich Wissenden isoliert, erwächst dann - wie gesagt (1.3. Anfang) - die Gegenkategorie: der Nicht-Fachmann, der Laie.

Etymologisch gesehen ist er der Massenmensch in seinem unspezifischen, grauen Alltag; denn engl. layman, franz. lä̈que, ital. und span. lä̈co gehen zurück auf griech. läós, attisch leös 'Volk', 'Menge'. Lat. lä̈cus verengt allerdings in kirchenlateinischer Zeit seine Bedeutung zu 'Nichtgeistlicher', dem somit der 'Geistliche' als Fachmann gegenüberstand. Für die Bedeutung 'Nichtkenner', 'Nichtfachmann' bot sich profanus an, und die Romania hat es mit franz. profane, ital. und span. profano im Sinne von 'ohne Kenner- und Könnerschaft' auch tradiert. Hier schei- 
nen nun die Merkmale des Verzäunens, des schützenden Geflechts, des abwehrenden Hegerings als deutliche kultursoziologische Gehalte aus historischer Zeit wieder durch; allerdings diesmal nicht vereinnahmend, als zum Fachlichen zugehörig (1.3.1.), sondern als Ausgrenzung, genau wie es profanus etymologisch angibt, nämlich als 'vor dem fanum', eben 'vor dem Heiligtum' verbleibend, als 'nicht geweiht', somit als 'nicht eingeweiht'.

Die sprach- und kulturgeschichtlichen Tatsachen belegen es also: Der Laie definiert sich negativ zum Fachmann; es ist nicht umgekehrt; vielmehr sind begrifflich der Fachmann und sein fachliches Können der Maßstab, an dem sich der Begriff des 'Laien' orientiert, wenn er - in negativer Bestimmung - mit 'Nichtfachmann' synonym gebraucht wird, wenn die Wörterbücher für 'Laie' angeben 'ungelehrt', 'etwas nicht gelernt habend', 'von etwas nichts verstehend', 'unvertraut mit einer Sache', 'nicht versiert', 'ohne Kenntnis'.

\subsection{Integration in einer Skala der 'Fachlichkeits'-Merkmale}

Diese Art zu definieren - und zwar negativ in bezug auf (!) eine gegebene Qualität ('fachlich') - erinnert uns zunächst an die Denkweisen, wie sie zuerst aus der strukturalen Phonologie bekannt geworden sind: In den dreiBiger Jahren entwickelte Roman Jakobson im Umkreis des Moskauer und später dann des Prager Linguistischen Zirkels die Theorie der binären Korrelation zwischen zwei sprachlichen Elementen. Demnach unterscheiden sich im Deutschen die Phoneme $/ p /$ und $/ b /$ in ihrer Eigenschaft (feature) ${ }^{18}$ der Stimmhaftigkeit; sie ist das principium comparationis zwischen den beiden korrelierten Phonemen. In bezug darauf ist /p/ [-stimmhaft] , /b/ [+stimmhaft] ; der Phonologe sagt auch: /p/ ist merkmallos ('unmarkiert'), /b/ ist merkmalhaltig ('markiert'). Analog zur Phonologie fand dieser strukturale Zugriff auch Eingang in die Morphologie der Grammatik sowie in die Lexikologie, wobei der streng binäre Rahmen der Phonologie aufgebrochen wurde. Wir können ihn auch noch weiter ausdehnen, und zwar auf die Beziehung der beiden

18 Jakobson (1974, S. 280) legt zu Recht Wert auf eine systematische Klarheit mit folgender Unterscheidung:

"1. das principium comparationis, die Oppositionseigenschaft (Wesenszug, feature) wie z.B. die phonologische Quantität, welche der Korrelation der Lang- und Kurzvokale zugrunde liegt, oder die grammatische Zeit, welche die Opposition von Präsens und Prăteritum bestimmt;

2. das Merkmal des einen der beiden Opposita, welches der Merkmallosigkeit des anderen Oppositum entgegengesetzt ist - wie etwa die Länge (Dehnbarkeit) der Kürze (Nicht-Dehnbarkeit) und die Vergangenheit der Nicht-Zeit des Präsens [...] ." 
Kategorien 'Fachmann versus Laie' zueinander:

Demnach wäre dann deren Beziehung geprägt von einer verbindenden Eigenschaft, nämlich dem Fachlichen, der Qualität der 'Fachlichkeit'. Die Kategorie 'Fachmann' ist dazu merkmalhaltig - oder sinnvoller gesagt: merkmalreich (zu ihrem Reichtum gehört auch die Opposition in 'handwerklich-praktisch' und 'wissenschaftlich-theoretisch'); und die zum 'Fachmann' oppositive, negative Kategorie 'Laie' ist dazu merkmalarm oder besser: merkmalreduziert.

Das traditionell gesehene Verhältnis von 'Fachlichkeit' und 'Laienschaft' hat sich in dieser Betrachtungsweise somit grundlegend verschoben: Gegründet auf den hier vorgestellten sachhistorischen und kulturgeschichtlich-etymologischen Erkenntnissen, liegt es danach nahe, über die Aufhebung der üblicherweise gehandhabten Gegenüberstellung nachzudenken: statt der Polarität der beiden Kategorien sollte ihre Vereinbarkeit gesehen werden. Das einigende Band ist die Eigenschaft oder der Wesenszug (feature) der 'Fachlichkeit'. Auf diese Weise steht nicht mehr in ungleicher Gewichtung dem einen Laie-Sein eine Palette vielfältiger Fachlichkeiten gegenüber. ${ }^{10}$ Vielmehr wird die Kategorie des 'Laien', des 'Laienhaften', der 'Laienschaft' von dem Merkmal der 'Fachlichkeit', das ja der Kategorie des 'Faches' und des 'Fachlichen' eigen ist, miterfaßt; und zwar als die merkmalreduzierte Form der Fachlichkeit. Dies läft es dann hinfällig werden, eine Gegensatzkategorie 'Laie' anzunehmen oder bestehen zu lassen; vielmehr wäre nun der kenntnisnegierende und alltagsorientierte Begriff der 'Laienschaft' in die Kategorie der 'Fachlichkeit' integriert: und zwar als deren Extrempunkt - oder besser noch: Endbereich -, was es nahelegt, die Qualität der 'Fachlichkeit' als gestuft, als auf einer Skala angeordnet aufzufassen. Am anderen Extrembereich dieser Skala wäre somit die "reine" Fachlichkeit, die vollkommen ausgewiesene Könnerschaft, das durch und durch sachorientierte Experte-Sein angesiedelt, eben als merkmalreichste Form der Fachlichkeit. Zwischen diesen beiden Extremen liegt die ganze Welt des zielgerichteten Handelns, in all ihrer Vielfalt und ihren Nuancen. Begriflich bieten die Sprachen hierzu sogar Orientierungsmarken, indem sie diese Stufung semantisch zum Ausdruck bringen: so in sehr feiner Differenzierung - die über Wortfeldanalysen und Kontextuntersuchungen herausgearbeitet werden müßte - Begriffe wie 'Professioneller', 'Experte', 'Spezialist', 'Kapazität', 'Fachmann', 'Meister seines Faches' und mögli-

10 Wie auch innerhalb der verschiedenen Fächer, also auf der Seite des Fachlichen, die gegenseitige Fachfremdheit nicht mehr von einem anderen Begriff von 'Laie' - nämlich zwei Fachleute (!) verschiedener Fächer sind füreinander Laien - durchzogen wird. Cf. Kalverkämper 1983, S. 143 f. 
che andere; oder mit deutlicherer Stufung 'Interessierter' ('Liebhaber einer Wissenschaft, eines Fachgebiets'), 'Dilettant' (dies sogar mit negativer Konnotation), 'kundiger Laie', 'Amateur' ('Liebhaberwissenschaftler'20 ); oder in der kompakten Abfolge von Nomenklaturen, die dem geltenden Ausbildungsweg 'vom Laien zum Fachmann', 'vom Alltag zur beruflichen Arbeit' folgt: so 'Schüler(in)' - 'Lehrling/Auszubildende(r)' oder 'Student(in)' - 'Geselle/Gesellin' - 'Meister(in)' - und andere. Über die jeweilige Position auf der 'Fachlichkeits'-Skala, auf die hin ein Handlungszusammenhang geortet werden kann, entscheidet dessen jeweiliger Reichtum oder jeweilige Armut an Merkmalen der Fachlichkeit.

\section{Sprachsystematische Gegensatz-Relation: Fachsprachlichkeit und Gemeinsprache}

\subsection{Gemeinsprache und Fachsprachen}

\subsubsection{Fächer und (ihre) Fachsprachen}

Ich will den gerade geäußerten Gedanken einer merkmalorientierten 'Fachlichkeits'-Skala anstelle einer Gegensatz-Beziehung zwischen 'Fach(lichkeit)' und 'Laientum' zu jenem Mittel hinwenden, das es überhaupt erst ermöglicht, Fächer zu erstellen, ihnen als Größen der Welt und des Handelns Inhalte und Ziele zu geben, ihre Grenzen zu umreißen oder festzulegen, ihre Verhältnisse zu Nachbarfächern zu klären - nämlich zur Sprache. Dafür sei unterstrichen: ${ }^{21}$ Die Einteilung der außersprachlichen Welt, von Ausschnitten aus der Lebenswirklichkeit, sagen wir: der Objekte, Sachverhalte und Handlungszusammenhänge als 'fachlich' ist nur

20 Der wissenschaftsgeschichtlich wichtigen und kulturhistorisch bereichernden Rolle des "Amateurismus" - einem (wie das Laie-Sein) noch unerforschten Komplex - widmen sich mit interessanten Beiträgen die „Berichte zur Wissenschaftsgeschichte" 9, 1986, Heft 3 u. 4. - Die Zwischenstellung zwischen 'Laie' und 'Fachmann' ist für den Curioso, Virtuoso, den Amateur, Liebhaber, Dilettant, Autodidakt, Privatgelehrten, Fachfremden, Außenseiter annäherungsweise markiert: ${ }_{n}$ der außerhalb institutionalisierter und etablierter Wissenschaft eine wissenschaftliche Leistung vollbringt, die in die etablierte Wissenschaft eingeht. Als Person kann er entweder außerhalb der wissenschaftlichen Profession bleiben, durch seine Leistung in sie aufgenommen oder zum Begründer einer neuen Wissenschaft oder wissenschaftlichen Disziplin werden" (Toellner 1986, S. 143). Zur (historischen) Ortung auf der Skala zwischen 'Laie' und 'Fachmann' bieten sich zunächst drei Aspekte an (loc.cit.): die ,individuelle Disposition" ("Sammler und Sucher, Erfinder und Entdecker, Grübler und Denker"), die "soziale Disposition" ("Stand und Stellung, Beruf und Tätigkeit, Geschlecht und Bildung"), die „prädisponierten Wissenschaften" ( inhaltliche oder methodische Novität, Attraktivität oder Utilität, Publizität oder Professionalisierungsgrad"). - Cf. auch Mann 1982.

Cf. Kalverkämper 1983, S. 130 f., 134, 137. 
durch Kommunizieren darüber, nur mit Hilfe der Sprache möglich. Es gibt keine 'natürlichen' Fächer, keine Fächer a priori. Es ist die Sprache, die ein Fach konstituiert und die ihm zur Konventionalisierung verhilft; und mit der allein es nur möglich ist, das Fach zu verändern in seinen Inhalten, Grenzen und Zuordnungen zu anderen Fächern (Hierarchien). Selbst der des öfteren getroffene Unterschied zwischen 'Beruf' als erwerbsmäßiger Arbeitsverrichtung und 'Fach' als umfassenderer, durchaus mehrere, auch heterogene Berufe übergreifender Kategorie ist nicht im Sachlichen begründet, sondern nur semantisch, also sprachlich bestimmt.

Um so weniger ist es dann einzusehen, daß sich bis in die jüngere Zeit hinein die Meinung hält, Fachsprachen seien analog zu den Fächern zu unterscheiden, ihre Anzahl orientiere sich an der Anzahl der Fächer. ${ }^{22}$ Hier ist nämlich die Perspektive falsch, nämlich vom Fach auf seine Fachsprache hin, gerichtet, statt umgekehrt die Konstituierung eines Faches von der Sprache - wir können sie Fachsprache nennen - abhängig zu machen. Zudem bietet eine solche Eins-zu-Eins-Relation zwischen Fach und seiner Fachsprache keinerlei sinnvollen Anhaltspunkt, wenn man, wie bereits angesprochen (1.2.1.), bedenkt, wie unsicher und relativ die Anzahl von Fächern überhaupt ist; sollen wir etwa tatsächlich 300 oder etliche tausend Fachsprachen oder selbst auch nur so viele Fachwortschätze annehmen?

\subsubsection{Laienwelt des Alltags und (ihre) Gemeinsprache}

Und wenn die Fächer ihre Fachsprachen haben, dann, so wird weiter aus der tradierten Vorstellung von den zwei außersprachlichen Kategorien 'Fachlichkeit' und 'Laienhaftigkeit' abgeleitet, muß auch die laienhafte "Wirkwelt" (Jost Trier) des Alltags ihre Sprache haben: nämlich die Gemeinsprache. Die traditionelle polare Ansicht hat sich somit von außen her auf die Kategorien der Sprachbeschreibung und Sprachgliederung aufgesetzt, was dazu führte, daB - analog zu 'Laientum' und 'Fachlichkeit' - eine prinzipielle Gegensätzlichkeit zwischen Gemeinsprache und Fachsprachen angenommen wurde.

\subsubsection{Modellvorstellung: sektoral und relational}

Sie prägte entscheidend die Modellvorstellungen über das Gefüge des Wortschatzes, an dem diese Gegensätzlichkeit ökonomischerweise, aber

22 Zum Beispiel Fluck 1985, S. 16; Möhn/Pelka 1984, S. 35; Wüster 1973. Die Diskussion hierzu wird unter dem Stichwort 'Horizontale Gliederung der Fachsprachen' geführt. Cf. auch Hoffmann 1985, S. 58-62. 
eben deshalb auch beschränkterweise illustriert wurde: Innerhalb von Kreismodellen ${ }^{23}$ wurde die Gemeinsprache als Zentrum angesetzt, um die sich dann in konzentrischen Ringen die nicht-gemeinsprachlichen, sprich: die fachlichen Wörter-Kategorien legen.

In den fünfziger Jahren, noch weit vor dem breiter aufkommenden Interesse an Fachsprachen in den endenden sechziger, in den siebziger Jahren, galt das sektorale Modell, wie ich es nennen möchte und es beispielhaft mit Kurt Baldingers Vorschlag (1952) ${ }^{24}$ repräsentiert sehe, als angemessene Strukturierung. Die Übergangsgürtel und Durchlässigkeiten der Sektoren (allerdings nur der hier im Modell miteinander benachbarten!) sind dann in den sechziger Jahren neu umgesetzt worden, indem sie als Austauschprozesse und als Übergangsbewegungen aufgefaßt und entsprechend gezeichnet wurden: Der Versuch von Werner Reinhardt $(1966),{ }^{25}$ der hier beispielhaft für Modelle angeführt sein soll, die ich als relationale Modelle bezeichnen möchte, trägt dieser erkannten Relativität mit konzentrischen und zentropetalen und zentrofugalen Pfeilen Rechnung. Zudem sind die Wortschatzringe selbst nicht mehr verabsolutierend (Baldinger), sondern vage und relativierend mit Größen wie 'stark' und 'wenig' gekennzeichnet. Die Opposition zwischen Gemeinsprache und Fachsprachen ist zwar durch Beziehungen gemildert, weil überbrückt (und zwar interessanterweise nur in einer Pfeilrichtung, nämlich von außen, von den Fachsprachen, nach innen, in die Gemeinsprache hinein, nicht auch umgekehrt), aber sie ist nicht aufgehoben.

Die Kategorie 'Gemeinsprache' ist gegenüber der in den Modellen vielfältig differenzierten Kategorie der 'Fachsprachen' eine zwar von diesen bedrängte, aber doch eine dort als homogen ausgewiesene und eigenständige Kategorie; sie ist dort eine zwar nur punktuelle, aber eine eben im 'Mittel'-Punkt angesiedelte Kategorie. Sie findet sich in den linguistischen Handbüchern, in den Sprachgeschichten, in den einschlägigen Arbeiten zur Lexikologie wie auch zur Lexikographie, selbst in den praxisorientierten Werken wie den Sprachlehrwerken und den Wörterbüchern (den sogenannten 'allgemeinen Wörterbüchern') als eine

23 Die Bevorzugung von Kreismodellen ist nicht nur gestaltpsychologisch interessant. Es mag auch die Evidenz dahinter stehen, die die von der Prager Schule Mitte der sechziger Jahre entwickelte Theorie von Zentrum und Peripherie des Sprachsystems für sich beanspruchen darf. Seibicke 1973, S. 96 bzw. 102 und - leicht verändert - in Baldinger 1982. Cf. Wolf 1979.

25 Abgedruckt auch in Heller 1970 sowie in Drozd/Seibicke 1973, S. 96 bzw. 102 und in Fluck 1985, S. 19. 
Klassifikationsgröße für die Sprache; ihrerseits wiederum leitet sie sich, wie gerade gesagt (2.1.2.), aus der außersprachlichen Kategorie des 'Laien' ab.

Dies ist eine arbeitspraktische, eine wissenssoziologische Rechtfertigung für die Kategorie 'Gemeinsprache' (1.1., 1.3.2.). Mit ihr hängen alle anderen Aspekte ursächlich zusammen, die man in Definitionsversuchen ausdrücklich oder in Vorannahmen implizit findet und die oft auch Konkurrenz- oder Modifikationsbezeichnungen für 'Gemeinsprache' zur Folge haben wie 'Allgemeinsprache', 'Alltagssprache', 'Einheitssprache', 'Standardsprache', 'Umgangssprache', '(öffentliche) Verkehrssprache' und andere. Grundsätzlich gemeint ist ,jenes Instrumentarium an sprachlichen Mitteln, über das alle Angehörigen einer Sprachgemeinschaft verfügen und das deshalb die sprachliche Verständigung zwischen ihnen möglich macht" (Hoffmann 1985, S. 48).

Es ist nun sicherlich müßig, hier noch weitere ähnlich lautende Definitionen und Beschreibungen im einzelnen vorzustellen; eine lange Tradition seit Grimm, die allseits bekannt sein dürfte, tut sich hier auf; wobei man allerdings sehen sollte, daß bei der Vätergeneration der Germanistik - so zum Beispiel im Grimmschen Wörterbuch (Grimm 1897, S. 3270) oder bei Hermann Paul (1880, Kap. 23) - die 'Gemeinsprache' als übergeordnete und damit, wie Paul sagt, als nicht reale, als ideale Kategorie über den Mundarten angesiedelt wird; ein Gegensatz zu Fachsprachen wurde so gar nicht gesehen. In diesem Verständnis, als ausdrücklicher Zusatz "ohne Mundarten oder Fachsprachen", wird 'Gemeinsprache' bis in die moderne Zeit geführt, so im "Duden-Wörterbuch der deutschen Sprache" (Duden 1980) oder im "Wörterbuch der deutschen Gegenwartssprache" (Klappenbach/Steinitz 1976).

\subsubsection{Merkmale der Gemeinsprache}

Aber weiter wiederkehrende Gesichtspunkte für die Definition von 'Gemeinsprache', stets eingeschränkt auf 'Gemeinwortschatz', sind in der Forschung zu denen der Anbindung an die Laien und der diatopischen, also mundartlichen Neutralität (2.1.2.1.) hinzugekommen: so die Frequenz - 'Gemeinsprache' als die häufigsten Wörter - sowie die Rekurrenz - als die am meisten wiederkehrenden Wörter (wozu natürlich auch der gesamte Morphembestand der Grammatik gehört) -; damit zusammenhängend auch der Umfang und Inhalt (Funktion) - 'Gemeinsprache' kommt vor im Alltag, im familiären Ablauf, im lockeren Verkehr mit den Mitmenschen, in zwangloser Unterhaltung, ist also allen Sprechern einer Sprachgemeinschaft eigen -; sowie, eng damit verbunden, die Anzahl der zugehörigen Sprachelemente. Dieser ist ein sehr vager Gesichtspunkt, da 
weder die Zählkriterien (ob Morpheme und Lexeme einzeln gezählt werden, wie Komposita zähltechnisch gehandhabt werden, usw.), noch die Begrenzungskriterien, noch die Auswahlkriterien diskutiert oder offengelegt sind. Hier wirkt sich das angesprochene Dilemma (1.1.) belastend aus, weder theoriegesichert noch praktisch am Einzelfall - und sei dies nur intuitiv - entscheiden zu können, was 'gemein', 'Alltag', 'nicht fachspezifisch' ist, und dementsprechend auch nicht das Sprachmaterial - hier nun die Wörter - als 'gemeinsprachlich' methodisch sauber einstufen zu können. Und so kommt es, daß die Statistiker Größenordnungen nennen von 500.000 (wie bei dem sechsbändigen Duden-Wörterbuch 1976-1981) über 400.000 und 300.000 Wörter, was verschiedene Linguisten vertreten und breiten Konsens findet, bis zu 220.000 Wörter (wie im sechsbändigen Brockhaus-Wahrig 1980-1984) und sogar nur 150.000 Wörter (wie im "Neues Wörterbuch" 1980 von Lutz Mackensen).

Der individuelle Aktiv-Allgemein-Wortschatz eines Erwachsenen wird auf 2.000 bis zu 20.000 Wörter, also auf durchschnittlich 10.000 Wörter geschätzt; der latente Aktivwortschatz dazu beläuft sich auf etwa 40.000 Wörter. Der individuelle Passiv-Allgemein-Wortschatz eines Erwachsenen soll angeblich 180.000 Wörter umfassen. Im Wechsel zu einer Fremdsprache schrumpft der Umfang an gemeinsprachlichem Bedarf ganz rapide: Etwa 2.000 fremdsprachliche Wörter für den aktiven Gebrauch und 8.000 für das Allgemeinverstehen sind errechnet worden. Hier bleibt dann natürlich zu fragen, wo die Gründe für eine derartige Verengung liegen und woraus sich, sollte sie tatsächlich zutreffen, eine solche Diskrepanz erklärt.

Diese Zahlen, die im einzelnen doch sehr vage bleiben und bei der Einschätzung eines Wortes als 'gemeinsprachlich' im jeweiligen Einzelfall überhaupt nicht weiterhelfen, dürften als Gesichtspunkt für eine nähere Bestimmung der Kategorie 'Gemeinsprache' wenig Wert, noch nicht ein$\mathrm{mal}$ einen Orientierungswert beanspruchen.

\subsubsection{Verständlichkeit und funktionale Modellvorstellung}

Schließlich gilt es, sich noch einem letzten Gesichtspunkt zu widmen, nämlich der für 'Gemeinsprachlichkeit' als unabdingbar deklarierten und deshalb für sie meist ausdrücklich genannten "allgemeinen" Verständlichkeit. Dies ist, im Gegensatz zu den anderen angeführten Gesichtspunkten für traditionell verstandene Gemeinsprachlichkeit, eine grundsätzlich relationale Qualität. Sie führt, wie die bereits genannte Einheit 'Kategorie' (1.3.), direkt zurück in die griechische und lateinische Antike, die die Verständlichkeit - perspicuitas - auffaft als eine Wort- und Textqualität - oder in den Worten der Antike: als eine stilisti- 
sche und rhetorische Tugend -, die an Sprecher und Hörer wechselseitig gebunden ist und nur in konkreten Kommunikationssituationen entschieden wird. ${ }^{26}$ Wir wissen, daß sich daraus für situationsenthobene - also: für schriftlich-literarische - Texte und für religiöse Traditionstexte dann zwangsläufig ein Suchen nach Verständlichkeit, also der Wunsch nach Interpretation, eine (literarische) Hermeneutik (und theologische Exegetik) also, entwickelt hat.

Gemeinsprache oder - wie es ja traditionellerweise praktisch immer gemeint ist - der gemeinsprachliche Wortschatz kann als solcher also gar nicht die absolute Qualität einer 'allgemeinen Verstāndlichkeit' innehaben - schon diese beiden kombinierten ganz relationalen Begriffe belegen die Unmöglichkeit -; und so kann sie erst recht nicht - als ein besonderes Charakteristikum - verständlicher sein als die Fachsprachen. 'Verständlichkeit' ist eine Textqualität, und als solche hat sie Gültigkeit für alle Arten von Texten und ist prinzipiell abhängig von den Beteiligten jeder funktionierenden Kommunikation: von Sprecher/Schreiber, Hörer/Leser, von der Sprache (als Texte oder in Texten) und von der jeweiligen Situation. Somit ist auch die vorangenommene 'Allgemeinheit' eine relationale Größe, die sich sowohl auf die sozialen Gruppen, Strukturen und Rollen bezieht, als auch auf eine wie auch immer geartete (geschweige erarbeitete) Situationen-Typologie. In der konkreten Kommunikation wird diese Relativität festgelegt, die 'Allgemeinheit' wird in der jeweiligen Situation mit den jeweils beteiligten Partnern zur Individualität, reduziert eben von Faktoren wie Alter, Geschlecht, Herkunft, Lebenslauf, soziokulturellem Hintergrund, Intelligenz, Lerngeschichte und Bildung oder Vorkenntnissen.

So verwundert es schon, daß gerade mit diesem komplexen Gesichtspunkt der textuell und sozial variablen Verständlichkeit der bislang letzte Versuch unternommen wurde, und zwar von Klaus Heller (1970), ${ }^{27}$ um das Verhältnis von Gemeinsprache - er verwendet den Ausdruck 'nicht fachbezogene Lexik' - und Fachlexik in ein Modell zu bringen. Ich bezeichne derartige Modelle als funktionale Modelle, da sie in die Kommunikations-

26 Quintilian 1972-1975, spez. 1,6,43; IV,2,36 u. 63-65; VIII,2; VIII,3,15. - Verarbeitet in Kalverkämper 1988a, 1988b; hier auch Anbindungen an die verschiedenen Wort- und Textqualitäten, die eine sich seit einiger Zeit zu Wort meldende Stilistik der Wissenschaftssprache, eine Fachsprachen-Stilistik, fordert (zum Beispiel Hans-Martin Gauger, Els Oksaar, Uwe Pörksen, Harald Weinrich); als Orientierung cf. Kalverkämper/Weinrich 1986, Teil II:

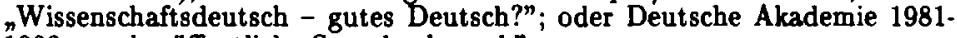
1982 zu „der öffentliche Sprachgebrauch”.

27 Abgedruckt auch in Drozd/Seibicke 1973, S. 96 bzw. 102 sowie in Fluck 1985 , S. 20. 
situation hineinweisen, aus der allein heraus entschieden wird über Graduierungen von Verständlichkeit und Spezialisierung, über Zugehörigkeit eines Wortes zu einem bestimmten Fachbereich, über den Status eines Wortes als Fremdwort oder als '„gemeinsprachlicher" Anglizismus' oder als 'fachlicher Anglizismus' oder als neues Wort oder weiteres.

Es muB allerdings gesagt werden, da $B$ mit einem funktionalen Modell allein noch nicht das Phänomen 'Gemeinsprache' systematisch erklärt, geschweige die Verhältnisse zwischen den Kategorien 'Gemeinsprache' und 'Fachsprachen' erfaßt sind. Das läßt sich auch nicht anders leisten als mit umfassenden empirischen Arbeiten. Und diese sollten induktiv vorgehen, so daB sie mit wenig belastender theoretischer Vorgeprägtheit die Texte - mündliche oder schriftliche, und aus möglichst vielfältigen Textsorten - auf ihre Fachsprachlichkeit hin untersuchen. Als Ballast wäre es dann anzusehen, wenn nun weiterhin von einer $Z$ weiteilung in 'Gemeinsprache' und 'Fachsprachen' ausgegangen würde:

\subsection{Beziehung von Gemeinsprache und Fachsprachen in referentiellen Texten}

\subsubsection{Konsequenzen gegen die Kategorie 'Gemeinsprache'}

Meine bisherigen kritischen Ausführungen dürften gezeigt haben, gegen wen des Gegensatzpaares sich der Vorstoß richtet: gegen die Kategorie der 'Gemeinsprache'. Sie hat sich schon terminologisch als Allerweltskategorie herausgestellt im Sinne von:

- darin findet sich alles auBer das Fachsprachliche;

- sie ist keinesfalls so homogen, wie es behauptet (zum Beispiel Drozd/Seibicke 1973, S. 86) und in Wortschatzmodellen dargestellt wird (2.1.2.1.);

- sie leistet nicht die Trennschärfe zu den Fachsprachen, die ihr mit einem Bündel angeblich charakteristischer Gesichtspunkte zugedacht wird (2.1.2.);

- sie ist in entscheidenden Punkten eine ausschließlich relativ bestimmte Größe, nämlich bei dem textuellen Merkmal der 'Verständlichkeit' (2.1.2.3.) und bei dem außersprachlichen Merkmal ihrer Gebundenheit an 'Laien' (2.1.2.);

- schließlich, aus diesem letzteren abgeleitet und im Einklang mit den vorigen gewichtigen Kritikpunkten, rechtfertigt sie ihre Existenz als linguistische Kategorie aus außersprachlichen Gegebenheiten, nämlich den Laien, heraus (2.1.2.), und diese sind, wie gesagt (1.4.), relativ ('Laie in bezug auf'), und sind, was ich eingangs entwickelt habe (1.3.2.), an den 'Fach'-Begriff gebunden. 
Eine faßbare sprachliche (!) Kategorie 'Gemeinsprache' kann es somit nicht geben, jedenfalls nicht in einer Seinsweise oder in einer Vorkommensart, die es gestattet, sie im Umfang zu begrenzen, sie inhaltlich zu präzisieren und sie in ihren Funktionen zu erkennen. Warum soll dann eine linguistische (!) Kategorie 'Gemeinsprache' eigenständig weiterbestehen bleiben, wenn sie doch für eine nicht vorhandene sprachliche (!) Kategorie keine systematischen, analytischen oder deskriptiven oder auch nur heuristischen Aufgaben erfüllen kann? Wenn für 'Gemeinsprache' die wissenschaftliche Definition keine Klarheit und Einheitlichkeit schafft, eben weil diese in der sprachlichen Wirklichkeit selbst nicht gegeben ist, sollte man konsequenterweise auf eine entsprechende Kategorie verzichten.

\subsubsection{Kritik an der Kategorie 'Gemeinsprache' in der Fachsprachen-Forschung}

Die erarbeitete Konsequenz, die sich gegen die Kategorie 'Gemeinsprache' wendet, kann - trotz der gemeinhin im (intuitiven) Konsens üblichen Gegenüberstellung von 'Gemeinsprache' hier und 'Fachsprachen' dort -. nicht ganz ungewöhnlich klingen. Gerade nach der ersten Phase der sich etablierenden Fachsprachen-Forschung, nämlich in der $z$ weiten Hälfte der siebziger Jahre, war schon ein kritisch ablehnendes BewuBtsein gekeimt:

\subsubsection{Sicht auf Sprachsystem: „Gesamtsprache”}

So betont Lothar Hoffmann in seinem Markstein setzenden Buch "Kommunikationsmittel Fachsprache" von 1976 zwar noch mit dem Blick des Sprachstatistikers insbesondere auf die Morpheme der einzelsprachlichen Grammatik, es treffe nicht zu, „daß die Gemeinsprache eine Fiktion wäre. Sie existiert; ohne sie wäre sprachliche Kommunikation nicht denkbar. Aber sie ist eine Abstraktion, wenn auch eine Abstraktion niederer Ordnung" (1985, S. 48; [1.A. 1976, S. 163] ). Aber schon aus der Beschäftigung mit den spezifischen Auffälligkeiten der Fachsprachen heraus wertet er dann später, daß nes keine allgemeinsprachliche Kommunikation gibt"' (1985, S. 127; [1.A. 1976, S. 263] ) und es theoretisch wie praktisch "kaum möglich" ist, die Zugehörigkeit von Sprachelementen zu einer vorangenommenen Gemeinsprachlichkeit zu bestimmen: "es [gibt] eigentlich keinen Platz mehr für gemeinsprachliche Kommunikationsakte" $\left(1985\right.$, S. 50; [1.A. 1976, S. 165] )..$^{28}$ Als Konsequenz

28 Die Aufhebung einer starren Gegenüberstellung von 'Gemeinsprache' und 'Fachsprachen' findet sich programmatisch wiederholt, wenngleich nicht als Problem entwickelt oder mit Lösungsangebot, in These 15.1 der "Leipziger Thesen zur fachsprachlichen Forschung" von 1977: Hoffmann 1988 [1977] , 
schlägt Hoffmann (1985, S. 51 f.; [1.A. 1976, S. 169]) vor, im übergeordneten Rahmen einer allumfassenden "Gesamtsprache" eine "möglichst vollständige Einteilung der gesamten sprachlichen Kommunikation in eine bestimmte Anzahl von Kommunikationsbereichen mit ihren jeweiligen Subsprachen" anzustreben.

\subsubsection{Sicht auf Textsorten und Sprachverwendungs-Situationen:}

Kommunikative Reichweite von Fachsprache

Im Jahre 1978 habe ich mich dann selbst aus textlinguistischer und textpragmatischer Sicht kritisch gegen die Annahme einer Kategorie 'Gemeinsprache' gewandt. ${ }^{2 \theta}$ Es wollte mir nicht einsichtig sein, woraus sich der immer wieder angenommene Primat der Gemeinsprache rechtfertigt, auf den hin die anderen Sprachvarietäten - hier die Fachsprachen - eingeschätzt und betrachtet werden: Als Gegenkategorie zu 'Fachsprachen' steht sie zu ihnen in einem völlig disproportionalen Verhältnis ('Eins' zu 'Vielfach und noch mehr'). Als eine Schnittmenge mit Merkmalen wie 'allgemein bekannt', 'alltäglich verwendet', 'hochfrequent', 'verständlich', 'Sprachbestand des Durchschnitts der Individuen einer Sprachgemeinschaft' (2.1.2.) müßte es eigentlich möglich sein, die Reichweite und die Inhalte - Quantität und Qualität also - der Gemeinsprache erfassen zu können. Aber genau dies läßt sich, wie aufgezeigt, weder im Sachlichen noch im Sprachlichen in der wünschenswerten Weise leisten. Da sind die Fachsprachen wesentlich präziser zu beschreiben und zu erfassen, was ihr Inventar, ihre sprachlichen Merkmale, ihre Funktion, ihren Bezug auf den außersprachlichen Referenten (als 'fachlich' eingestufte Objekte, Sachverhalte und Handlungszusammenhänge), ihre Verwender (Fachleute) betrifft. Es sind eben nicht die Grenzen der Gemeinsprache in einem Sprachmodell, die den Beginn der fachsprachlichen Zone bestimmen - ein solches Modell ist schon in seiner gestalterischen Anlage falsch bedacht. Denn es ist ein steriles Modell isoliert genommener Lexik, das die kommunikative Dimension außer acht läßt und somit die Reichweite des Sprechens „for special purposes" (Kalverkämper 1978, S. 431) nicht sieht. Eingebrachte Grauzonen, Übergangsgürtel, Austauschfelder sind für das Selbstverständnis solcher Modelle nur lästig, weil in der Abstraktheit des Modells vage und unscharf. Stattdessen - so war (und ist) das Votum aus textpragmatischer Sicht - sind die Unsicherheiten in der $\mathrm{Zu}$ weisung gemeinsprachlicher oder fachsprachlicher Qualität neu zu interpretieren, und zwar als kommunikative Chancen (Kalverkämper 1979, S.

S. 21 .

Kalverkämper 1978, 1979, 1980a. 
$65 \mathrm{f.}$ ): als Residuen im fachsprachlichen Kommunizieren (Kalverkämper 1982, S. 112-117), als Entscheidungen des (Fach-)Autors in Hinblick auf seinen Rezipienten ('Fachsprachen-Hermeneutik': Kalverkämper 1988). Nicht die Grenzen und die Übergänge - so die Schlußfolgerung daraus -, also nicht ausschlieBlich die Eigenständigkeiten von Fachsprachen und ihre Vermischung mit der angeblichen Gemeinsprache sind vorrangig weiterführende Ziele linguistischer Forschung, sondern die kommunikative Reichweite von Fachsprache, ihre Einbettung in Texte-in-Funktionen: Aus dieser Position heraus ergeben sich dann konsequent jene Fragen, die zeigen, wie brüchig die Gegenüberstellung von 'Gemeinsprache' und 'Fachsprachen' ist und wie wenig die konzessionsbereite Annahme von Mischungen und Übergängen an Erklärungs- und Bestimmungswert bietet: Ist - zum Beispiel - das Wort Hammer gemeinsprachlich oder fachsprachlich? Oder das Wort - und es handelt sich ja in den bisherigen methodischen Zuweisungen stets um wortbezogene Entscheidungen - Fünfzylinder, Satellitenübertragung, Sweat Shirt? Oder ist - zum Beispiel - das Gespräch einer Hausfrau mit einer Nachbarin über die angemessene Wahl von Pflegemitteln für die Terrasse mit Marmorboden gemeinsprachlich? Oder fachsprachlich? Ist es fachlich (sachbezogen), aber nicht fachsprachlich? (Kalverkämper 1978, S. 425-427). Oder, als weiteres Beispiel, sind Zweck- oder Gebrauchstexte wie Reiseprospekte, Nachrichtensendungen, Polizeiberichte, Reportagen, Spielanleitungen usw. fachsprachlich oder fachsprachlich-gemeinsprachlich vermischt oder gemeinsprachlich-fachsprachlich durchsetzt oder überhaupt nur gemeinsprachlich? ${ }^{30}$ Gezielte Textanalysen ${ }^{31}$ in diesem Fragenkreis der Uneindeutigkeit von Zuweisungen als traditionellerweise 'gemeinsprachlich' oder 'fachsprachlich' haben es nahegelegt, neben der Konsequenz, daf man bei dem üblichen Gegensatzpaar auf die 'Gemeinsprache' verzichten sollte, jenen Versuch einer methodischen Umsetzung zu entwickeln, den ich im späteren vorstellen möchte (3.).

\subsubsection{Sicht auf Varietäten: „Alltagssprache”}

Zunächst aber sei noch der Überblick mit einem weiteren Befürworter einer "Abschaffung des Ausdrucks "Gemeinsprache" als Teil eines komplementären Begriffspaares zur Beschreibung sprachlicher Systeme" (Hartmann 1980, S. 32) abgeschlossen: Dietrich Hartmann (1980) setzt als Leitorientierung den 'Varietäten'-Begriff ein, der insbesondere

${ }^{30}$ Kalverkämper 1978, S. 438-442; 1979, S. 67 f.; 1980a, S. 13-16; 1982; 1983, S. $142 \cdot 157$.

31 Kalverkämper 1982, 1987, 1988a, 1990. 
die mitschwingende Auffassung vom Sprachsystem als einem homogenen Ganzen ${ }^{32}$ unterlaufen soll. Danach unterscheidet er die schriftlichen und mündlichen Varietäten der Gesamtsprache nach fachsprachlichen und nach alltagssprachlichen ${ }^{33}$ Varietäten $(1980$, S. 34). Der Begriff 'Gemeinsprache' ist somit verschwunden, und die neue Kategorie - situationsspezifisch-alltagssprachlich - findet ihren Stellenwert innerhalb eines sprachtheoretischen Rahmens. Es ist selbstverständlich, daß die Kategorie der Varietäten - neben den situationsspezifischen noch die sozialen und (bei den mündlichen) die regionalen Varietäten - enge Kontaktnahmen und Interferenzen untereinander nahelegt. Deshalb sind für einen solchen Ansatz gerade die Einflüsse - fachsprachliche auf alltagssprachliche - von besonderem Interesse. Vor dem Hintergrund eines allgemein noch dürftigen Forschungszugriffs und einer bislang nur sehr schmal erarbeiteten Erkenntnislage setzt sich Hartmann (1980, S. 37-42) für verstärkte empirische Untersuchungen und vorrangige methodische Zielsetzungen ein. ${ }^{34}$

32 So zum Beispiel bei Drozd/Seibicke 1973, S. 86. Allerdings hat es bei der anstehenden Problematik - Gemeinsprache gegenüber Fachsprachen - ein solches Homogenitätsprinzip für die textpragmatische Sichtweise schon per definitionem nicht gegeben: Eindeutig Kalverkämper 1982, S. 105-109, HessLüttich 1987, S. 177-201.

33 Mit der Ersetzung von 'gemein-' durch 'alltags-' ist zunächst einmal nicht viel gewonnen; die bekannten außersprachlichen Schwierigkeiten werden durch neue ersetzt: siehe 1.1 .

34 Der breite Aufgabenkatalog programmatischer Arbeitsperspektiven für die Fachsprachen-Forschung, den Hartmann in seiner Einschätzung zusammenstellt, steht nicht im Einklang mit jener Einschätzung Lothar Hoffmanns (1988 [1982] , S. 34), nach der bis zum Ende der achtziger Jahre alle prinzipiellen Fragen der Fachsprachen-Forschung angesprochen und im wesentlichen geklärt sein sollen. Auch nach meiner Meinung gibt es noch zu viele Aporien, intuitive Entscheidungen und methodologische Probleme - von denen eines der wichtigsten und grundlegenden der vorliegende Beitrag behandelt -, um sich aus linguistischer Sicht bereits zufriedengeben $\mathrm{zu}$ wollen. Diese Sichtweise findet auch darin ihre pragmatische Bestätigung, daß in der längeren letzten Zeit neue Aufgabenbereiche auf die FachsprachenForschung zugekommen sind; ich denke an die neue semiotische Ausrichtung einer "Fachsprachen-Onomastik", wie sie, im Rahmen der Leipziger Schule, von Rosemarie Gläser und Mitarbeitern betrieben wird (zum Beispiel Gläser 1986); ich denke an Weiterentwicklungen im Rahmen der Terminologiearbeit, die auf neue Herausforderungen von Gesellschaft und Technologie reagieren (Überblick zum Beispiel bei Felber/Budin 1989); ich denke weiterhin an Bemühungen, die kognitiven Prozesse zu erarbeiten, wie sie sich bei der intensiven Einbindung von Sachgebieten in die verschiedenen Gruppen der Fachleute und Sachwissenschaftler ergeben und bestimmend auf Sprechen und Handeln einwirken; ich denke ferner an jene Ansprüche, die von außen an die Fachsprachen-Forschung und ihre Erkenntnisse gestellt werden, so bei der Technology Accessment-Forschung (Technikfolgen-Abschätzung), so bei dem gesellschaftlich sehr relevanten Problemkreis um 'Verständlich- 


\section{Integration in einer Skala der 'Fachsprachlichkeits'-Merk- male}

\subsection{Chancen und Perspektiven}

\subsubsection{Traditionelle Methode: Sammeln und Zuweisen}

Was die Handbücher, Sprachgeschichten, Wörterbücher und Grammatiken zum Verhältnis von 'Gemeinsprache' und Fachsprachen bieten, gleicht bislang - gerade auch wegen der sprachtheoretisch noch unbefriedigenden Methodik und der analysepraktisch noch bescheidenen empirischen Grundlagen - mehr oder weniger umfangreichen Mustersammlungen, Wörterauflistungen und Zusammenstellungen von (meist lexikalischen) Beispielen. Dies sei mit allem Respekt festgestellt; denn es bedarf zu solchen linguistischen Kärrnerarbeiten geduldigen Beobachtens und Zusammentragens durchaus jener ausgeprägter Tugenden, die Harald Weinrich (1976, S. 347) bei den Sammlern und Ordnenden ausmachte.

Allerdings, so meine ich, kann es nicht wissenschaftlich zufriedenstellen, allein festzuhalten, daß es Fachgebiete gibt, „von deren Handeln heutzutage fast jeder, unmittelbar oder mittelbar, betroffen ist (z.B. Verkehrstechnik, Haushaltsgerätetechnik, Postwesen, Bankwesen, Steuerwesen, Medizin, Datenverarbeitungstechnik) oder für deren Handeln ein breites Interesse besteht (zum Beispiel Sport, Fotografie, Fernsehen, Touristik) bzw. ein starkes Interesse beansprucht wird (zum Beispiel Raumfahrt, Kerntechnik, Informatik, Videotechnik)" (Möhn/Pelka 1984, S. 142); wozu dann Beispiele als mehr oder weniger reichhaltige, prinzipiell offene Aufzählungen von isolierten Wörtern geboten werden.

Neben solchen sachlich-fachlich bedingten Anreicherungen der 'Gemeinsprache' werden auch die Metaphorisierungen von Fachwörtern im gemeinsprachlichen Gebrauch der privaten und öffentlichen Alltagskommunikation angeführt und dann mit umfangreichen Korpora nach den bildspendenden Fachgebieten abgesteckt: das sind heutzutage besonders die Fächer 'Technik', 'Naturwissenschaften', 'Wirtschaft(swissenschaften)', 'Sport' und 'Militär'.

keit' und die damit verbundenen Gesichtspunkte von Verständnis (Bildung, Allgemeinbildung) und Verständigung (soziale Interkommunikation) (Kalverkämper 1988b), so auch bei der Sprachvermittlung in allen Perspektiven gerade in letzter Zeit mit wachsendem Interesse bei 'Deutsch als Fremdsprache' -, wo die Fach- und Wissenschaftssprachen wegen des neuen Kommunikationsbedarfs immer stärker in das Interesse der Didaktik und Methodik und Sprachenpolitik rücken. 


\subsubsection{Ausweg: Skala der 'Fachsprachlichkeits'-Merkmale}

Doch so sehr man im einzelnen Forschungsbeitrag einer derartigen Methodik zu folgen bereit sein mag, sollte man doch prinzipiell kritisch bedenken: Wen der Einfluß der Fachsprachen auf die sogenannte 'Gemeinsprache' interessiert - und dies ist, wie Weinrich (1976b, S. 368) sagt, ,ein zentrales Problem unserer Gesellschaft" -, kann sich nicht damit zufriedengeben, Wörter zu sammeln und Fächer im Konsumalltag zu orten. Schon die verbreitete Weise, solche Entwicklungen zu beschreiben, verstellt den Blick auf die eigentlichen Phänomene, die sich da vollziehen: Es trifft eben nicht zu, daß sich 'Fachwörter in die Gemeinsprache hineindrängen', daß sie 'hinüberwandern' und 'sich ansiedeln'.35 Solche Anthropomorphismen sind typische Fälle von „Deagentivierung”, deren Wirken und Wirkung Peter von Polenz (1981) sprachkritisch untersucht hat. Sie täuschen eine Bewegung, eine Aktivität vor, die auf jene Aufzählungen von Wörtern abfärben soll, die der Lexikograph oder sprachbeschreibende Autor nur intuitiv erkundet, nur subjektiv als solche Wanderwörter eingeschätzt hat. ${ }^{36}$

Sucht man aber nach dem eigentlichen 'Agens' solcher "Beeinflussungen” oder "Wanderungen", so findet sich der konkrete jeweilige Text, es finden sich die Kommunikationspartner, zwischen denen er ausgetauscht wird, es findet sich die umfassende Kommunikationssituation. Wenn wir tatsächlich den jeweiligen, den konkreten Sprachgebrauch berücksichtigen würden und methodisch beachteten und dazu die traditionelle Kategorie 'Gemeinsprache', wie vorgestellt (2.2.), als Gegenkategorie zu den Fachsprachen beiseite ließen, dann könnte sich die Parallelität zu jenen Überlegungen offenbaren, die ich bereits zu den außersprachlichen Kategorien 'Fachlichkeit' und 'Laienschaft' angestellt habe (1.3.): Dort war ja der Begriff des 'Laienhaften' mit guten Gründen in den des 'Fachlichen' überführt worden, und zwar als dessen (skalenartig gestuft-) merkmalreduzierte Form.

Und nun auch hier, im Bereich des Sprachlichen, sollte man den komplementären Begriff zu 'Laienschaft' und 'Alltag', nämlich die 'Gemeinsprache', dementsprechend aufheben und ihn - ganz parallel zu den me-

35 Cf. entsprechende Kritik in umfassenderem Zusammenhang bei Kalverkämper 1982, S. 115-117; 1983, S. 145-147. Cf. 3.2.2., Punkt 3.

36 Bei dieser Sichtweise bleibt als eine Herausforderung die Frage, wieviel fachlichen Bezug denn ein Wort verloren haben muß, wieviel Fachsprachlichkeit es abgeben muß, um als 'gemeinsprachlich' gelten zu können; oder kann es sogar die Qualität der 'Gemeinsprachlichkeit' bei so viel vorhergegangenem 'Verlust' dann als einen 'Gewinn' verbuchen? Cf. 3.2.2., Punkt 3. 
thodologischen Überlegungen zum Außersprachlichen ('Fach[lichkeit] ') - in den Partnerbegriff, also die Fachsprachlichkeit, integrieren. Demnach wäre alles Kommunizieren über die Gegenstände, Sachverhalte und Handlungszusammenhänge - kurz: über "die Welt" - als fachsprachlich anzusehen, und zwar ebenfalls in gestufter - hier nun: sprachlicher Merkmalhaftigkeit. Streng fachliches Kommunizieren zwischen Fachleuten wäre somit äußerst merkmalreich, ein Gespräch von Hausfrau zu Hausfrau über die angemessene Waschmitteldosierung oder eine Nachfrage und Antwort zum richtigen Zeitabstand beim Füttern von Jodperlen für den Wellensittich wären merkmalarm.

\subsection{3. 'Fachsprachlichkeit' als Eigenschaft der Skala}

\subsubsection{1. 'Fachsprachlichkeit' als Orientierung}

Die grundsätzliche Eigenschaft (feature, 1.4.) der Skala ist die 'Fachsprachlichkeit', wie sie sich in den einzelnen Texten der Lebenspraxis (zwischen "Alltag und Beruf") verdeutlicht; und zwar in gestufter Weise zwischen den Polen '(extrem) merkmalreich' und '(extrem) merkmalarm' ${ }^{37}$ Das tertium comparationis ist damit zugunsten der 'Fachsprachlichkeit' festgelegt; von ihr aus bestimmt sich jene Sprache-inTexten, die bislang intuitiv als 'Gemeinsprache' eingeschätzt wurde, als (merkmal-)arm, als (merkmal-)reduziert, also als Verringerung derjenigen Merkmale, die in Fachsprachen reich, vielfältig, komplex, auffälig vorhanden sind. Die Perspektive bei der Besetzung der Skala mit Merkmalen verläuft, da es sich um (gestufte) Merkmale der 'Fachsprachlichkeit' handelt, somit in einer Richtung: von den Fachsprachen als Orientierungsbereich hin zu der sogenannten 'Gemeinsprache'. Diese Sichtweise rechtfertigt sich aus etlichen Gründen:

1. Zunächst - und schon argumentationsmächtig genug - sind die kulturgeschichtlichen Tatsachen ins Gedächtnis zu rufen (1.1., 1.3.1.): der sachbezogen-fachlich-berufliche Handlungsraum als primäre "Wirkwelt" des intentional tätigen Menschen. Ihr Bild hat sich im Laufe der Kultur-, Sozial- und Bildungsgeschichte seit den Anfängen der Menschheit, dann, besser greifbar, seit Altertum und Mittelalter beständig gewandelt; eine noch im 17. Jahrhundert recht einheitliche Arbeitswelt hat sich inzwischen mit neuen oder veränderten Wissensgebieten, Arbeitsbereichen und Tätigkeitsfeldern zu einer arbeitsteiligen, hochspezialisierten,

37 Eine sprachliche Qualität 'merkmallos' gibt es in Texten-in-Funktion genauso wenig, wie es die außersprachliche Qualität der 'Nicht-Fachlichkeit' in Situationen mit Bezug auf Gegenstände, Sachverhalte oder als Handlungszusammenhänge nicht gibt. (Bestes Beispiel ist hierzu der eingangs erwähnte 'Alltag': 1.1.). 
„expertokratischen” Lebensweise entwickelt. Das hat im 18. Jahrhundert dazu geführt, daf sich ein Begriff von 'Fach' herauskristallisiert, dem sich ein Begriff von 'Alltag' zugesellt, der sich allerdings - wie das Spektrum nalltäglicher" Tåtigkeiten der modernen Zeit belegt (1.1.) - entgegen den üblichen Einschätzungen durchaus nicht als 'unfachlich' konturiert.

2. In diesem Sinne ist zudem daran zu erinnern, daß das Laie-Sein ebenfalls aus der Sicht des Fachmanns begrifflich erfaßt wird (1.3.2.), nämlich per negationem ('Nicht-Fachmann') und eben nicht eigenwertig positiv. In Situationen, in denen das Wissensgefälle - auch dieser Begriff ist negativ-perspektivisch vom Fachwissen aus besetzt - typisch ist, nämlich in Lernsituationen, zeigt sich, „daB dem kleinen Kind die Sprache der Erwachsenen als eine Fachsprache entgegentritt" (Weinrich 1976d, S. 110); Weinrich folgert daraus, "daß alle Lernprozesse" - und was könnte oder soltte nicht hierunter fallen, wo doch, wie gesehen (1.1.), selbst der Alltag sich als ein solcher entpuppt hat - „über die Aneignung einer Fachsprache laufen" (loc.cit.).

3. Als eine ausgreifende Sichtweise ist begrifflich und terminologisch nur die eine Richtung 'von dem Fach aus hin zur Allgemeinheit', 'von der Fachsprache aus hin zur sogenannten Gemeinsprache' erfaßt: nämlich als 'fach-extern', als 'popularisieren' (lat. populum!), 'vulgarisieren' (lat. vulgus!), 'laiisieren'. Für die Gegenrichtung gibt es nicht derartige kompakte Begriffe.

4. Hiermit zusammenhängend verstehen sich auch die Ansprüche der gesellschaftlichen Gruppen untereinander in einseitiger Richtung: von den Kenntnisreichen, den Fachleuten, Experten, Spezialisten hin zu den Kenntnisarmen, den Uneingeweihten. Der hierbei verwendete Begriff 'Transfer' trifft genau diese Richtung der Vermittlung von Wissen und Fertigkeiten; der vergleichbare Begriff vom 'Wissensgefalle', dem kein komplementärer für die andere Richtung gegenübersteht (s. Punkt 2.), läßt ebenfalls keinen $Z$ weifel offen, daß es die Fachleute sind, die auf der exponierten, der "reichen" Stelle angesiedelt sind, von der aus alles andere tiefer, arm, 'außerhalb', 'vor dem Eingeweihten' liegend 'profan' im angesprochenen Sinn (1.3.2.) - erscheint. Aus der Sorge um eine funktionierende demokratische Gesellschaft heraus, in der 'Wissen' eine sozial kontrollierte Macht bleiben muß und das Verständnis der (fachlichen) Sache durch eine Verständlichkeit der Sprache gewährleistet ist, hat sich seit Mitte der siebziger Jahre die griffige Formel von der „Bringschuld" etabliert. Auch hierbei ist die einseitig gerichtete Aktivität, die Handlungsinitiative, die Macht des Auswählens deutlich mitgemeint: Die Fachleute sollen sich, in sozialer Verantwortung und in wissenschaftsethisch begründetem Anspruch an sich selbst, dem interessierten, 
engagierten Laienpublikum, dem mündigen und aufgeweckten Bürger gegenüber öffnen. Hierzu hat sich dann etliche Zeit später ein Gegenbegriff tatsächlich eingestellt: nämlich die „Holschuld", die diese fachinteressierte Öffentlichkeit ihrerseits bei den kooperationsbereiten Fachleuten - insbesondere den Fachwissenschaftlern - einlösen muß. Aus dem einseitigen 'Gefälle' mit Orientierung am Fachmann könnte sich somit ein gesellschaftlicher Dialog unter Partnern entwickeln. ${ }^{38}$

5. Für die merkmalorientierte Skala das $\mathrm{Maß}$ an der Fachsprachlichkeit - und eben nicht an der sogenannten 'Gemeinsprache' - zu orientieren, rechtfertigt sich auch aus der inzwischen anerkannten sprachsystematischen Tatsache, daß die Fachsprachen und die sogenannte 'Gemeinsprache' nicht, wie ehemals angenommen, eigenständige Charakteristika aufweisen, vielmehr die Fachsprachen ihre Mittel durch Abwahl, durch Selektion aus dem „Gesamtbestand der Sprache" (Hoffmann 1985, S. 47) gewinnen und für ihre speziellen $Z$ wecke in besonderer Weise bereithalten und einsetzen. Diese Sprachmittel in ihrer textgebundenen Gemeinschaft signalisieren 'Fachsprachlichkeit', sie markieren die fachbezogene Funktion des Textes und der Kommunikationssituation, sie bestimmen mit ihrem gestuften Merkmalreichtum die Einschätzung des Textes als (mehr oder weniger) Fachtext. ${ }^{39}$ Daraus rechtfertigt sich dann auch die Beobachtung, daß "sich das Inventar einer Fachsprache dank der Begrenzung ihres Wirkungsbereiches leichter vollständig erfassen läßt" es ist eben markierter ${ }^{40}$ und somit auffallig - als das der sogenannten 'Gemeinsprache', bei der ja "nicht eindeutig feststeht, in welchen kommunikativen Bereichen oder Situationen davon Gebrauch gemacht wird"

$38 \mathrm{Zu}$ diesem Umkreis Kalverkämper/Weinrich 1986: insbes. S. 163-168, Kalverkämper $1988 \mathrm{~b}$.

39 Recht augenfällig werden solche Merkmale dann, wenn sie als Mittel der Parodie eingesetzt werden: Eine besondere Tradition der Text-Variation hat das Märchen vom Rotkäppchen (Ritz 1986): Rotkäppchen „auf Mathematisch", "auf Theologisch", "auf Linguistisch", "aus der Sicht eines Chemikers", nauf Amtsdeutsch"; zu letzterem eine Kostprobe von Thaddäus Troll: "Im Kinderanfall unserer Stadtgemeinde ist eine hierorts wohnhafte, noch unbeschulte Minderjährige aktenkundig, welche durch ibre unübliche Kopfbekleidung gewohnheitsrechtlich Rotkäppchen genannt zu werden pflegt. Der Mutter besagter R. wurde seitens ihrer Mutter ein Schreiben zustellig gemacht, in welchem dieselbe Mitteilung ihrer Krankheit und Pflegebedürftigkeit machte, worauf die Mutter der $R$. dieser die Auflage machte, der Großmutter eine Sendung von Nahrungs- und Genußmitteln zu Genesungszwecken zuzustellen. [...]." (loc.cit., S. 106).

40 Und diese Markiertheit bezieht sich auf pragmatisch-sachliche, soziologische, situative, sprachliche und textsortenspezifische Merkmale. 


\section{(Hoffmann 1985, S. 52). ${ }^{41}$}

6. Und noch ein weiteres gewichtiges, ein textuelles Argument soll es rechtfertigen, daß als Maßstab für eine integrierende Skala die (gestufte) Fachsprachlichkeit gewählt wird: nämlich das der Vielfalt an fachlichen (besser: fachsprachlichen) Textsorten (Gattungen). Man sollte sich die Frage beantworten, welche Textsorten im Konsens als tatsächlich sogenannte 'gemeinsprachliche' gelten können. Aufgezählt werden - so bei einer Straßenbefragung und bei einer Fragebogenaktion ${ }^{42}$ - 'Alltagsgespräch', 'Reden in der Familie', 'Unterhaltung', 'Plauderei', 'Schwätzchen', 'Gequatsche'; nur sind diese Textvorkommen nicht Angehörige von Textsorten, sondern sie sind Kommunikationsarten. ${ }^{43}$ Sie lassen sich nämlich nicht mit den fünf textexternen Faktoren vollständig erfassen, die zur Konstituierung von 'Textsorten' - neben einer Hierarchie textinterner Faktoren - vonnöten sind:44 'Sprachliche Grundfunktion' (Ausdruck, Darstellung, Appell), 'Typ von KommunikationsprozeB' (alltäglich, öffentlich/rechtlich, wissenschaftlich, literarisch), 'Bereich der Gegenstände und Sachverhalte' (Raum- und Zeitbezug), 'Gemeinsame Kommunikationssituation zwischen Sprecher und Hörer' (vorhanden, teilweise vorhanden, verschieden), 'Kommunikationsrichtung' (Monolog, Dialog). Dementsprechend läßt sich auch die ${ }_{n}$ kommunikative

41 In diesem Sinne auch Weinrich (1976c, S. 36) im Umkreis der Austinschen Sprechakttheorie: "Wenn [...] wohl für immer unklar bleiben wird, wo in der Gemeinsprache die Grenzen des Versprechens liegen, so ist doch in der Sprache des Rechts immer schon festgelegt, was ein Heiratsversprechen ist und was nicht. Auch bei der Taufzeremonie, um auf Austins Leitbeispiel zurückzukommen, ist durch das Kanonische Recht genau bestimmt, welche Formen ein Taufgelöbnis haben muß, um gültig zu sein. Die im Bürgerlichen oder Kanonischen Recht fixierten Gültigkeitsregeln geben dem Sprechakt des Versprechens jene scharfen Konturen, die er in der Umgangssprache eben nicht hat."

42 Siehe Anmerkung 7.

43 Wie „Telekommunikation, Rundfunksendung, Buch, Zeitungsartikel” (Gülich/Raible 1975, S. 155) - Oder die Ausprägungen von phatischer Kommunikation - homileische Diskurse, Small talk - im Hausflur, über den Gartenzaun hinweg, im Omnibus usw., die die (durchaus wichtige) Funktion haben, soziale Kontakte sprachlich zu ermöglichen und aufrecht zu erhalten.

44 Cf. Gülich/Raible (1975, S. 145): „Textvorkommen, die intuitiv als Manifestationen bestimmter Textsorten angesehen werden, die aber nicht durch alle Gruppen, sondern nur durch eine Teilmenge der Gruppen textexterner Merkmale zu beschreiben sind, sind keine Manifestationen von Textsorten, sondern Kommunikationsarten."

Von den ersten drei Merkmalgruppen sind sie bei zweien nicht spezifizierbar (loc.cit., S. 155): bei "sprachliche Grundfunktion" und bei "Bereich der Gegenstände und Sachverhalte". 
Funktion"45 sogenannter 'gemeinsprachlicher' „alltäglicher" Textvorkommen nicht festlegen. Und überhaupt konvergieren die fünf semiotischen Qualitäten, die für Textsorten bzw. (literarische) Gattungen gelten, ${ }^{46}$ nicht im Lebensraum des Alltags, nicht im Kommunikationsumfeld der sogenannten 'Gemeinsprache': Wie gerade über die textexternen Faktoren gesehen, leistet es die (a) "Kommunikationssituation" nicht, eine gemeinsprachliche Textsorte zu ndeterminieren"; und es gibt keine (rhetorische, sprachnormierende, formale) "Konvention” (b) des Vertextens; über den (c) ${ }_{n}$ Komplexitätsgrad" läßt sich wegen fehlender Rahmenvorgaben nichts aussagen; eine (d) „Abgeschlossenheit” läßt sich mangels Begrenzungskriterien und hierarchischer Ordnungen nicht feststellen; die Interpretation der sogenannten 'alltäglichen Kommunikation' in der sogenannten 'Gemeinsprache' ist, da es keine vorsteuernde Rezeptions- (und Produktions-) Richtlinien gibt, durchaus nicht einer (e) „Einschränkung" unterworfen.

Ganz anders dagegen im fachlichen Handeln und fachsprachlichen Kom-

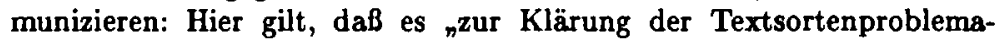
tik kein günstigeres Terrain als das der Fachsprachen" gibt (Hoffmann 1988 [1982] , S. 26). Thr Reichtum an unterschiedlichsten Zweck- oder Gebrauchs- oder expositorischen Texten ist außerordentlich; in ihnen offenbart sich die eng ineinander greifende Beziehung von Handeln und Sprechen, von Sprache-in-Funktion, oder noch spezieller: von Fachsprache (oder Fachtexten)-in-Funktion: Anleitung, Gutachten, Lehrbuch, Protokoll, Rezension, Kommentar, Zeugnis, Rundschreiben, Bewerbung, Reklamation, Urteil, Vertrag, Anordnung, Gebrauchsanweisung, Handbuch, Lohnzettel, Fertigungsvorschrift, Stellenausschreibung, Rezept, Patentschrift, Schadensmeldung, Nachrichten, Wetterbericht, usw. ${ }^{47}$

Vor diesem Hintergrund wird es nicht verwundern, daß die fachsprachlichen Texte, eben nicht die - sollte man eine solche Kategorie überhaupt annehmen - 'gemeinsprachlichen' Texte und ebenso auch nicht die literarischen Texte, ein bestimmender Faktor in der Sprach- und Textegeschichte sind: „Das Fachschrifttum war das weitaus meistverbreitete und das heißt meistgelesene Schrifttum in jenen Jahrhunderten,

45 Cf. Gülich/Raible (1975, S. 146, 155 (Zitat] ): „Die Spezifizierung eines Textvorkommens nach allen fünf Gruppen textexterner Merkmale ergibt die sogenannte ${ }_{n}$ kommunikative Funktion" des Textvorkommens."

46 Ich orientiere mich mit den fünf Punkten (a) bis (e) an Raible 1980, S. 324-338.

47 Cf. zum Beispiel Fluck 1985, S. 207-214, Gläser 1979, S. 83-178, Möhn/Pelka 1984, S. 71-128. 
als die Schriftsprache entstand" (Eis 1967, S. 55) und übte wegen des wesentlich breiteren Lesepublikums aller "Stände vom Kaiserhof bis zur Schäferhütte" (loc.cit.) - Rezipienten der höfischen Dichtung waren ja auf den Adelsstand beschränkt - eine machtvolle Wirkung aus. Es dürfte sich somit auch aus der Perspektive der Textetradition rechtfertigen, die Fachsprachlichkeit als jenes $\mathrm{MaB}$ zu wählen, das die gestufte Skala, die die Gegensatzpole 'Gemeinsprache' und 'Fachsprachen' integrativ auflösen soll, bestimmt.

\subsubsection{Die gleitende Skala}

Die Vorstellung von einer gleitenden Skala soll also die traditionelle Meinung von den Antipoden ablösen. Auch verkappte Dichotomien gehören dann nicht hierhin: so die Lösung von Heller (1970) oder von Drozd/Seibicke (1973, S. 82 f.), einfach die theoretischen Aporien und die auch im Praktischen nicht lösbaren Schwierigkeiten dadurch umgehen zu wollen, daß - betrachtend von den recht gut zu sichernden Fachsprachen (3.1.3.1., Punkt 5.) aus - der Rest als „Nichtfachsprache" gilt. Damit ist $\mathrm{ja}$, bis auf eine terminologische Stringenz hin zu 'positiv fachsprachlich' und 'negativ fachsprachlich', kaum etwas gewonnen. Auch der Ersatz von 'gemeinsprachlich' durch 'alltagssprachlich' (Hartmann 1980) bringt kaum Vorteile (2.2.2.3.). Die störende Gegensatz-Relation bleibt bestehen.

Schon ein aufmerksames Betrachten der sogenannten Alltagskommunikation (zum Beispiel Hannappel/Melenk 1984) bestätigt, daß es angemessener ist, ein skalar sich verschiebendes Anreichern oder Abnehmen von Merkmalen der Fachsprachlichkeit festzustellen, wenn über Objekte, Sachverhalte und Handlungszusammenhänge gesprochen wird, also wenn die Kommunikation referentiell gewichtet ist: ${ }^{\mathbf{4 8}}$

48 Das ist auch die traditionelle „Domäne”, wenn das übliche Paar 'Gemeinsprache' gegenüber 'Fachsprachen' bemüht wird. - Mit dem Hinweis auf die referentielle Funktion (Symbolfunktion) ist also nicht vergessen oder gar bestritten, daB in der Kommunikation auch andere Funktionen - die expressive (Symptom) und die appellative (Signal), wenn man Karl Bühler folgt (1965, S. 24-33) - verwirklicht werden; die 'Sprache der Liebenden', das 'Gespräch mit der Telephonseelsorge', das 'Betteln um ein bestimmtes Geburtstagsgeschenk' - sie sind nur verschwindend gering referentiell gerichtet und gehören nicht in jenen Spannungsbereich zwischen - traditionell gesprochen - Gemein- und Fachsprache. DaB es auch hierbei Übergänge gibt, mag sich gerade an jenen expressiven und appellativen Kommunikationssituationen zeigen, auf die die Gesellschaft oder bestimmte Gruppen mit organisierendem Zugriff, mit Konventionen und Normvorgaben Einfluß ausüben: So zum Beispiel bei der 'Sprache des Gebets', 'Sprache der Kondolenz', das 'Sich Entschuldigen' und andere. - Cf. auch Steger (1988) zur Komplexität der "Binnengliederung" von Sprachsystemen - aus der ich also, 
Von der einfachen, fachsprachlich extrem merkmalarmen Frage Welche Kamera haben Sie da? kann sich da ein Gespräch über Spiegelreflex und Filmqualitäten bis hin zur Optik entwickeln; von der Aussage Das Weißbrot schmeckt hier nicht. kommt man über die Brotsorten zur Backkultur bis hin zu Ernährungsgewohnheiten und ihre physiologischmedizinische Einschätzung; der erstaunte Ausruf Sie lesen ja Umberto Eco! ist geradezu angelegt auf ein Weiterspinnen des Fadens in die fachliche Dichtigkeit der Literaturkritik und die Diskussion der neueren literarischen Strömungen; das Bedauern über eine Daumenverletzung kann das Gespräch auf das Thema Gesundheit allgemein bringen und es weiter mit dem Sprechen über die medizinische Versorgung bis hin zur Forschung anreichern; ein Sprung im Geschirr mag Anlaß dazu sein, sich über Vor- und Nachteile von Porzellan gegenüber Steingut auszutauschen und im Gespräch über die qualitativen Vorzüge und formgebenden Besonderheiten verschiedener Porzellanmanufakturen noch fachlicher zu werden bis hin zur Mikrowellentauglichkeit. Die Hausrenovierung, der Heizöleinkauf, das "sachgemäße", „fachgerechte" Prüfen von Kiwi-Früchten im Obstladen - sie sind wie jede andere Situation Anlaß dazu, die begleitende Kommunikation fachsprachlich zu verdichten. Es hängt an den Möglichkeiten und den Interessenlagen der Kommunikationspartner in ihrer Situation, wo dieser ProzeB jeweils endet. Daraus läßt sich die Einsicht ableiten, daß sich die referentielle Kommunikation zwischen fachsprachlich (extrem) merkmalarm über fachsprachlich merkmalangereichert zu fachsprachlich (extrem) merkmalreich verdichtet, und - in anderer Richtung - sie sich auflockert. Ein skalares Gleiten ist somit die angemessene modellhafte Vorstellung. Sie ermöglicht es dann auch, Beanstandungen zu relativieren, die sich auf möglicherweise nicht stimmige Zuweisungen von Wörtern oder syntaktischen Strukturen zu 'gemeinsprachlich' oder zu 'fachsprachlich' beziehen: Denn vielfach ich zitiere trotz der Anthropomorphismen (3.1.2.) - ,ist es ohne weiteres nicht ersichtlich, ob ein sprachliches Element aus der Fachsprache in die Alltagssprache oder von dort in die Fachsprache bzw. mehrere Fachsprachen gewechselt ist" (Hartmann 1980, S. 46, Anm. 26). So sieht von Hahn (1981, S. 4) den „Verdacht verstärkt, daß die Grammatikographie lange Zeit, durch nicht ausdrücklich oder nicht genau genug vorgenommene Korpusdefinitionen, eine ganze Zahl fachsprachlicher Strukturen (etwa Funktionsverbgefüge) fälschlich als gemeinsprachlich ausgewiesen hat". 49

nochmals betont, nur die Ordnungsbegriffe 'Gemeinsprache' und 'Fachsprachen' beachte und als Erscheinungsformen integrativ in Beziehung setze, andere mögliche also außer acht lasse.

40 Ähnlich skeptisch in von Hahn 1983, S. $111 \mathrm{f}$. 
Korpora und empirische Analysen (2.2.2.) - diese dürften in der Tat der geeignete Schlüssel sein, ${ }^{50}$ um Zugang zu einer nicht-polarisierten Auffassung zu erlangen. „Alle fruchtlosen Bemühungen festzustellen, welche Mittel für die Fachsprachen allein charakteristisch sind und welche sie der Gemeinsprache entlehnen, hätten ein Ende" (Hoffmann 1985, S. 49). Die Lösung sehe ich darin, eine skalar geordnete 'Fachsprachlichkeit' von referentiellen Texten-in-Funktion anzunehmen (2.2.2.2.). Dies würde helfen, den bislang noch recht starren Blick auf das Sprachsystem und seine innere Organisation (2.1.2.) zu lösen; bereichernd sollte sich dies für Analysen im Bereich der Sprachverwendung auswirken, wie es ja schon aus textpragmatischer Sicht gefordert wurde und für die FachsprachenForschung umgesetzt worden ist. ${ }^{61}$

\subsection{Instrumente und Methode}

\subsubsection{Merkmale der Fachsprachlichkeit: Vom Wort zum Text-in-Funktion}

Die bisherige Wissenschaftsgeschichte der Fachsprachen-Forschung umfalt inzwischen - rechnet man ziemlich eigenständig gebliebene Versuche als "Wirtschaftslinguistik" in den dreißiger Jahren (zum Beispiel Ewald E.J. Messing) nicht hinzu - etwa zwanzig, fünfundzwanzig Jahre. Sie sind von Anfang an geprägt von dem Bestreben, die Charakteristika der Fachsprachen zu erkennen: Zunächst auf der Ebene der Lexeme (Termini), dann der Syntax, später - Ende der siebziger Jahre - auf der Ebene der Texte, und inzwischen, mit den achtziger Jahren, in der Komplexität der fachsprachlichen Kommunikation überhaupt (Fachtext-in-Funktion, Kommunikationsverfahren). Methodisch sind dazu verschiedene Ansätze entwickelt und Modelle erstellt worden: funktionalstilistische, statistische, normende, strukturalistische, textpragmatische Wege werden in-

60 Ich habe dies in meinen Arbeiten zur Fachsprachen-Forschung stets gefordert (und an konkreten Textanalysen vorgeführt): speziell in Kalverkämper 1980a, 1982, 1983, 1987. Eine pauschale Meinung, wie sie von Hahn (1983, S. 3,119 f.) vorträgt, es gebe lediglich ein "Forschungs-Fordern", nicht ein "Forschung-Machen" in der Fachsprachen-Literatur, erst recht, was die Textstrukturen betreffe (S. 119 f.), läßt sich mit einer tieferen Kenntnis der tatsächlichen Forschungssituation - auch zu jener Zeit! - als ungerechtfertigt widerlegen.

61 Kalverkämper 1983 mit „Fach-Text-in-Funktion", Herausarbeiten der Vernetzung in Relationen, Bestimmen von Fachtext-Linguistik als "sortenbezogene Sprachverwendungs-Linguistik". Hoffmann (1988 [1985] ) stellt konsequent die Fachsprachen-Forschung in den wissenschaftssystematischen Rahmen der Angewandten Sprachwissenschaft. 
zwischen angeboten. Was sie an Erkenntnissen erbracht haben, mag im einzelnen nicht immer 'typisch' oder 'eindeutig' oder 'sicher' fachsprachlich sein; Gründe wie Schwächen in der Methodik, ihren Prämissen, Inkonsequenzen bei der Analyse, Fehlen einer empirischen Basis oder Schmalheit des Korpus mögen durchaus Unsicherheiten oder Ungenauigkeiten geschaffen haben. Aber gemeinschaftlich genommen liegt dennoch ein umfangreiches und in sich komplexes Arsenal an Ergebnissen zur Spezifik von Fachsprachen vor, das inzwischen über alle Ebenen der Sprachbeschreibung - von den Graphemen und Phonemen über die Lexik und Syntax zum Text und der Kommunikationssituation - reicht. Ich darf hier diese Merkmale der Fachsprachlichkeit als inzwischen breitest bekannt voraussetzen, zumal sie sich in den einschlägigen Handbüchern und Überblicksbeiträgen der Fachsprachen-Forschung zusammengestellt finden. Schlaglichtartig und um die Vielfalt und Signalkraft der Merkmale anzudeuten, seien hier nur einige Auffälligkeiten angeführt: ${ }^{52}$

- Terminologisierung, Internationalismen

- Komposition, Derivation

- Kasus-Vitalität (Genitiv in den deutschen Wissenschaftssprachen!)

- Funktionsverbgefüge

- Passiv-Bevorzugung

- Partizip, Infinitiv, Gerundiv

- Tempus-Dominanz (Präsens)

- Personen-Wahl (3. Person)

- Relationsadjektive

- Nominalisierung

- Deagentivierung

- Thema-Rhema-Verteilungen

- Entropie

- Textbaupläne (Makrostrukturen)

- Textstrukturen, Textkohärenzen, Textgliederungen (Teilganze, Hierarchien, Einbettungstiefen)

- Textsorten-Spektrum

- Situationsbindungen (Textpragmatik)

- Meta-Informationen (Reflexivităt)

- Text-Bild-Relationen.

Im Bereich des Wortschatzes ist die fachsprachliche Verdichtung durch Anreicherung entsprechender (lexikalisch-) fachsprachlicher Merkmale

52 Ich beschränke mich, um keinen bibliographischen Apparat aufzubauen, auf orientierende Werke: Drozd/Seibicke 1979, Felber/Budin 1989, Fluck 1985, bes. S. 47-59, Gläser 1979, von Hahn 1983, S. 83-126, Hoffmann 1985, Kalverkämper 1983. 
gerade mit kontrastiven Arbeiten - die noch von einer Dualität 'Gemeinsprache' versus 'Fachsprache' ausgehen - herausgefiltert worden. ${ }^{53}$ Die dabei gewonnenen Erkenntnisse legen es allerdings geradezu nahe, eher an eine skalenartige Organisation von fach(sprach)lichen Merkmalen zu denken, als zwei Erscheinungsweisen - wie dort noch als 'gemeinsprachlich' und 'fachsprachlich' (geographisch, technisch, u.a.) - zu verabsolutieren. Denn ohne die Kontexte, in die derartige Wörter eingebettet sind, verbleiben die Zuweisungen im Bereich der Intuition, des EvidenzAngebots (2.2.2.2.; 3.1.2.). So erkennt Große (1979), der ausgeht vom Modell „der Bildung neuer Fachsprachen, die sich satellitenartig um das Corpus der Gemeinsprache ansiedeln" (S. 209), daB für das einzelne Wort "die Zuordnung zu einer Fachsprache schwer[fallt] ": „Soll man ein Wort wie Krise der Philosophie, der Medizin, der Politik oder der Wirtschaft zuordnen; wohin gehört Formel: zur Mathematik oder zur Chemie? Oder sind beide Wörter überhaupt nicht den fachsprachlichen Lexika zuzuordnen?" (S. 213). Genau dies sind die Fragen, die das methodologische Dilemma aufzeigen (2.2.2.2.): die „Abgrenzungsschwierigkeiten, die jedem begegnen, der versucht, zwischen Fach- und Gemeinsprache zu unterscheiden" (Große 1979, S. 213). Der methodisch allein geeignete Raum für derartige Untersuchungen, die ja auch von Tragweite für die Lexikographie $^{54}$ und für die Sprachdidaktik, ${ }^{55}$ also für Ziele der Angewandten Linguistik, sind, können nur die Texte und ihre Kommunikationssituationen sein. Nur in Texten-in-Funktion zeigen sich die Merkmale der Fachsprachlichkeit, ${ }^{66}$ und $z$ war in ihrer jeweils gestuft vorkommenden

53 Ich denke zum Beispiel an Ischreyt (1965, spez. S. 231-253): „Genormte Terminologie in der Gemeinsprache"; oder an die eindrucksvolle "Studie von Hard (1970), die bislang keine vergleichbaren Fortsetzer gefunden hat (was wohl nicht zuletzt daran liegt, daB die hohe Zeit onomasiologischer und begriffsgeschichtlicher Fragestellungen - die ausgehenden fünfziger und sechziger Jahre - erst einmal verblaft ist). Als interessante Themen böten sich hierzu auch aktuelle Leitbegriffe wie 'Fach', 'Beruf', 'Prestige', 'Arbeit', 'Bildung', 'Kultur', 'Freizeit', 'Alltag' und andere an (nicht ganz in diesem Sinne des Oszillierens zwischen 'gemein-' und 'fachsprachlich' $\mathrm{cf}$. aber zum Beispiel: Europäische Schlüsselwôrter 1964-1967).

54 Als Hinweis hier nur Mentrup 1978a und Wiegand 1988.

55 Als Hinweis nur Wimmer 1979, Hoffmann 1988: Kap. IV. - Das Gesagte gilt auch für den Aspekt 'Deutsch als Fremdsprache', der sich immer stärker zu 'Fachdeutsch als Fremdsprache' differenziert; die DaF-Lehrwerke müssen auf den inzwischen drängend spürbaren Bedarf reagieren, wohlweislich erkennend, daß Fachsprachen "eine der wichtigsten Erscheinungsformen der deutschen Sprache unter dem Gesichtspunkt des Unterrichts sind" (Ickler 1984, S. 28); cf. auch Beier/Möhn 1984, Buhlmann 1982.

56 Orientierend seien nur angegeben Gläser 1988, Hoffmann 1988, Kalverkämper 1983, Möhn/Pelka 1984. 
Determinationskraft von '(extrem) merkmalarm' bis hin zu '(extrem) merkmalreich'.

\subsubsection{Methodischer Gewinn}

Die angestellten Überlegungen haben in das Spannungsfeld vom Wort zum Text-in-Funktion geführt, das sich als eine nach Komplexitätsgraden gestufte Skala beschreiben läßt. Indem es nun in ein weiteres Spannungsfeld - zwischen der sogenannten 'Gemeinsprache' und den Fachsprachen - eingebracht werden sollte, wurde zugleich dessen Aufösung deutlich: In der Gegensatzbeziehung zwischen 'Gemeinsprache' und Fachsprachen hat sich - gerade für referentielle Texte - die Kategorie 'Gemeinsprache' als eine nicht taugliche offenbart. Daraus lassen sich nun für die fachsprachenlinguistische Methodik vier Gesichtspunkte als Gewinn verbuchen:

1. Es sind nicht mehr vier Kategorien - nämlich 'Fach(lichkeit)' und 'Laie(nschaft)' sowie 'Fachsprache(n)' und 'Gemeinsprache' -, sondern nur noch zwei Kategorien gegeben: nämlich einerseits das außersprachliche Phänomen 'Fach' (mit seiner ihm jeweils eigenen arbeitsweltlichen bzw. wissenschaftlichen oder technologischen Qualität 'Fachlichkeit'); und andererseits das sprachliche Phänomen 'Fachsprache(n)' (mit den jeweils zugehörig signalisierten Qualitäten der 'Fachsprachlichkeit'). - Dies ist ein Gewinn für die Beschreibungsökonomie.

2. Die fachsprachlichen Signale sind an den jeweiligen Text gebunden. In ihm entscheidet sich, welche Möglichkeiten - von '(extrem) merkmalreich' bis zu '(extrem) merkmalarm' - umgesetzt worden sind. Zugleich ist dadurch der Produzent des Textes mit einbezogen, der fachsprachlich gestuft-merkmalreich oder -merkmalreduziert seinen Text verfaßt hat; und auch der Textrezipient ist miterfaßt, wie ihn der Autor mit seinem Text und den in ihm verwirklichten Signalen der Fach(sprach)lichkeit angezielt, ihn in seinen Verstehensmöglichkeiten antizipiert hat. ${ }^{57}$ - Diese textpragmatische Sichtweise, die sich abkehrt von einer starr systematischen Einteilung mit begrenzten Gegensatzkategorien (s. Punkt 1.), ist ein Gewinn für die Beschreibungskomplexität.

\section{Schematisierung der Fakten von 1. und 2.}

Die Gegensatzverhältnisse (Punkt 1.) und ihre Aufösung in graduell

57 Ich verweise hierzu nochmals auf das Konzept einer 'FachsprachenHermeneutik': Kalverkämper 1988a; zu dem gerade angesprochenen Zusammenwirken siehe dort auch die neuorientierende Konzeption von 'Verstehen' und 'Nicht-Verstehen' von (Fach-)Texten in fachexterner, also auf Laien bezogener Kommunikation (speziell S. 161-165, insbes. S. 164). 
gestufte Skalen (Punkt 2.) lassen sich in ihren gegenseitigen Bezügen schematisch darstellen:
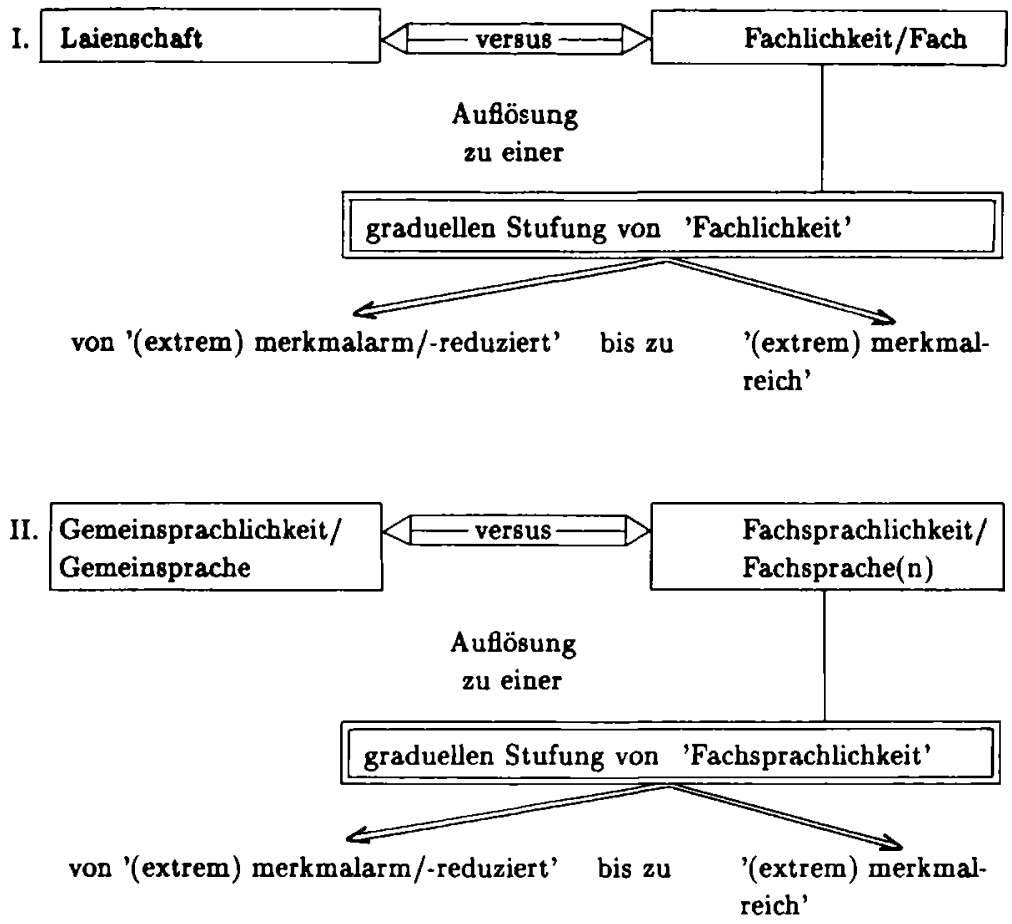

3. Die Zuweisung eines Wortes im traditionell gesehenen Spannungsfeld zwischen der sogenannten 'Gemeinsprache' und den Fachsprachen (hier dann: einer bestimmten Fachsprache) geschieht nicht mehr intuitiv, sondern nunmehr angemessen, weil über die textuell verankerten sprachlichen Merkmale beschreibbar, aus den Texten-in-Funktion ableitbar. Es mag ja immerhin schon der Widerspruch verwundern, der darin steckt, wenn üblicherweise von 'Fachwörtern in der Gemeinsprache' geredet wird: Wenn sie nämlich als in der 'Gemeinsprache' „angesiedelt" (3.1.2.) eingestuft werden, dann können sie ja wohl kaum mehr Fachwörter, sondern eben 'Gemeinsprache'wörter sein - wie auch immer man dies ermessen will; wenn sie aber als Fachwörter weiterhin identifiziert werden, können sie nicht als in die sogenannte 'Gemeinsprache' „hinübergewechselte" und dort heimische eingeschätzt werden. In dem größeren Rahmen der Frage nach der Einflußnahme von Fachsprachen auf die 
sogenannte 'Gemeinsprache' besagt dies konkret: Welche Eigenschaften muß ein Fachwort oder eine fachsprachliche Struktur in der sogenannten 'Gemeinsprache' noch oder wieder oder neu innehaben, um als "EinfluB" erkannt werden zu können? Man kann sich hierzu nicht auf philologische Beobachtungen, auf historische Betrachtungen, auf möglicherweise sogar etymologische Bemühungen berufen. Diese sind methodisch diesem Problem nicht gewachsen, wie die bisherigen Arbeitsweisen und Wege der Zuweisung belegen - gerade sie sind ja auch Zielpunkte der Kritik des vorliegenden Beitrags. - Die Hinwendung zum Text-in-Funktion und seinen sprachlichen (sowie anderen semiotischen - außersprachlichen - ) Signalen einer (gestuften) Fach(sprach)lichkeit, die die "Mischungsverhältnisse" von 'Gemein-' und Fachsprachlichkeit genauso erfaBt wie die "reine" Fachsprachlichkeit der jeweiligen Texte, ist ein Gewinn für die Beschreibungsstringenz.

4. Wenn man die beiden Kategorien der (außersprachlichen) 'Fachlichkeit' und der (sprachlichen) 'Fachsprachlichkeit' mit ihren Skalen von '(extrem) merkmalreich' bis zu '(extrem) merkmalarm' miteinander in Beziehung setzt, ergeben sich Korrelationen, die sich als textsortenrelevant, vielleicht als textsortenspezifisch herausstellen können:

Fachlichkeit

Fachsprachlichkeit
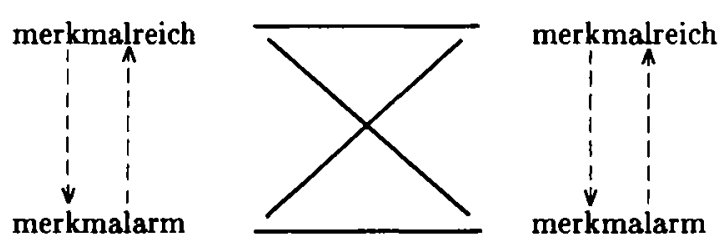

So könnte ein fachlich merkmalreicher Handlungszusammenhang, zum Beispiel der Zusammenbau eines Motors, fachsprachlich merkmalreduziert vertextet sein, was sich je nach Stufungsgrad als Vorlesung an der Technischen Hochschule, als Lehrbuchtext für Fünftsemester oder aber für Examenskandidaten, als Einführung für interessierte Kinder und Schüler ausweisen würde. - Dies ist ein Gewinn für die Beschreibungsklassifikation.

\section{Konsequenzen aus 3 . und 4.}

Ich habe mit meinen Überlegungen, die aus der Kritik an der traditio- 
nellen Gegensatz-Relation erwachsen sind und sich für eine integrierende Methodik einsetzen, die dazu notwendigen Instrumentarien vorgestellt:

- Zum einen die Merkmale der Fachsprachlichkeit: 3.1.3.;

- zum anderen die Ordnung der Merkmale als Skala: 3.1.2.

Was noch der Lösung harrt, ist die Hierarchie der Merkmale auf der Skala. Wieviele (a) und welche Merkmale (b) müssen im Text gegeben sein (c), um ihn als fachsprachlich merkmalreich, als einen Fachtext, erkennbar werden zu lassen? Wie sind die Merkmale im Text verteilt (d)? Lassen sich Korrelationen zwischen Quantität (a'), Qualität (b'), Rekurrenz ( $c^{\prime}$ ) und Distribution ( $d^{\prime}$ ) erkennen und als Hierarchie auswerten? An der Beantwortung dieser Fragen muß noch (weiter) gearbeitet werden. Daß sie beantwortbar sind, macht schon die tägliche Erfahrung mit jeglicher Art von Texten deutlich; sie setzt jeden einigermaßen sensiblen Kommunikanten instand, recht souverän darüber entscheiden zu können, ob in einer gegebenen Situation dieser Text "mehr" fachlich "wirkt", jener Text dagegen weniger fachsprachlich ist. Zunächst gilt es wohl, die absehbare Komplexität in den Griff zu bekommen und eine texte- und textsortenbezogene Bestandsaufnahme der relevant beteiligten Merkmale anzustreben - im Sinne der alten Logik, die die vorkommenden Unschärfen über kombinatorische Definitionen (die Form der definitio per proprietates) auszugleichen versuchte und im Idealfall sogar zur definitorischen Präzision führte.

\section{Literatur}

Arbeitsgruppe Bielefelder Soziologen (Hrsg.) (1973): Alltagswissen, Interaktion und gesellschaftliche Wirklichkeit. 1: Symbolischer Interaktionismus und Ethnomethodologie. - 2: Ethnotheorie und Ethnographie des Sprechens. Wiesbaden. (5. Aufl. 1981).

Baldinger, Kurt (1952): Über die Gestaltung des wissenschaftlichen Wörterbuchs. In: Romanistisches Jahrbuch 5, S. 65-94.

Baldinger, Kurt (1982): Séance de clôture: Le Colloque dans le cadre de la lexicologie historique du français. In: Höfler, Manfred (Ed.): La lexicographie française du XVIe au XVIIIe siècle. Wolfenbüttel, S. 149-158.

Beier, Rudolf/Möhn, Dieter (1984): Fachtexte in fachsprachlichen Lehr- und Lernmaterialien für den fremdsprachlichen Unterricht - Überlegungen zu ihrer Beschreibung und Bewertung. In: Special Language / Fachsprache 6, S. 89-115.

Bühler, Karl (1965): Sprachtheorie. Die Darstellungsfunktion der Sprache. Mit einem Geleitwort von Friedrich Kainz. 2. unveränd. Aufl. Stuttgart. (1. 
Bublmann, Rosemarie (1982): Analyse und Beurteilung fachsprachlicher Lehrwerke: Kriterien und ihre Problematik. In: Krumm, Hans-Jürgen (Hrsg.): Lehrwerkforschung - Lehrwerkkritik. Deutsch als Fremdsprache. München, S. 122-164. (Werkstattgespräche [Goethe-Institut, München] ).

Cicourel, Aaron Victor (1964): Method and Measurement in Sociology. New York. (Dt. Übers.: Methode und Messung in der Soziologie. Frankfurt 1970).

Dahlberg, Ingetraut (1974): Grundlagen universaler Wissensordnung. Probleme und Möglichkeiten eines universalen Klassifikationssystems des Wissens. Pullach bei München. (DGD [Deutsche Gesellschaft für Dokumentation] - Schriftenreihe. 3).

Deutsche Akademie für Sprache und Dichtung [Darmstadt] (1980-1982): Der öffentliche Sprachgebrauch. Stuttgart. I: Die Sprachnorm-Diskussion in Presse, Hörfunk und Fernsehen. Bearb. v. Birgitta Mogge. 1980. - II. Die Sprache des Rechts und der Verwaltung. Bearb. v. Ingulf Radtke. 1981. - III: Schulen für einen guten Sprachgebrauch. Bearb. v. Birgitta Mogge/Ingulf Radtke. 1982.

Drozd, Ludomir/Seibicke, Wilfried (1973): Deutsche Fach- und Wissenschaftssprache. Bestandsaufnahme - Theorie - Geschichte. Wiesbaden.

Duden (1980): Das große Wörterbuch der deutschen Sprache in sechs Bänden. Hrsg. u. bearb. vom Wissenschaftlichen Rat und den Mitarbeitern der Dudenredaktion unter Leitung von Günther Drosdowski. MannheimWien-Zürich.

Eis, Gerhard (1967): Mittelalterliche Fachliteratur. 2. durchges. Auf. Stuttgart. (Realienbücher für Germanisten. Abt. D: Literaturgeschichte).

Elias, Norbert (1978): Zum Begriff des Alltags. In: Hammerich/Klein (1978), S. 22-29.

Europäische Schlüsseiwörter (1964-1967): Europäische Schlüsselwörter. Wörtervergleichende und wortgeschichtliche Studien. Hrsg. v. Sprachwissenschaftlichen Colloquium (Bonn). München. - II: Kurzmonographien, I: Wörter im geistigen und sozialen Raum. 1964. - III: Kultur und Zivilisation. 1967.

Faulseit, Dieter (1975): Das Fachwort in unserem Sprachalltag. Leipzig.

Felber, Helmut/Budin, Gerhard (1989): Terminologie in Theorie und Praxis. Tübingen. (Forum für Fachsprschen-Forschung. 9). 
Fluck, Hans-Rüdiger (1985): Fachsprachen. Einführung und Bibliographie. 3. aktual. u. erw. AuA. Tübingen.

Garfinkel, Harold (1967): Studies in Ethnomethodologie. Englewood Cliffs (N.J.).

Gläser, Rosemarie (1979): Fachstile des Englischen. Leipzig. (Linguistische Studien).

Gläser, Rosemarie (Hrsg.) (1986): Eigenname und Terminus. Beiträge zur Fachsprachenonomastik. Leipzig. (Namenkundliche Informationen [Karl-Marx-Universität] . Beiheft. 9).

Gläser, Rosemarie (Hrsg.) (1988): Fachtext als Instrument und Resultat kommunikativer Tätigkeit. Leipzig. (Berichte der Sektion Fremdsprachen [Karl-Marx-Universität] . 3).

Goffman, Erving (1974): Frame Analysis: An Essay on the Organization of Experience. New York. - (Dt. Übers.: Rahmen-Analyse. Ein Versuch über die Organisation von Alltagserfahrungen. Frankfurt 1977).

Grimm, Jacob/Grimm, Wilhelm (1897): Deutsches Wörterbuch. 4. Band, 1. Abteilung, 2. Teil. Leipzig.

Grosse, Siegfried (1979): Beobachtungen zum fachsprachlichen Vokabular im Leitartikel einer Tageszeitung. In: Mentrup (1979), S. 209-217.

Gülich, Elisabeth/Raible, Wolfgang (1975): Textsorten-Probleme. In: Linguistische Probleme der Textanalyse. Jahrbuch 1973 (des Instituts für deutsche Sprache, Mannheim] . Düsseldorf. (Sprache der Gegenwart. 35), S. 144-197.

von Hahn, Walther (Hrsg.) (1981): Fachsprachen. Darmstadt. (Wege der Forschung. 498).

von Hahn, Walther (1983): Fachkommunikation. Entwicklung - Linguistische Konzepte - Betriebliche Beispiele. Berlin-New York. (Sammlung Göschen. 2223).

Hammerich, Kurt/Klein, Michael (Hrsg.) (1978): Materialien zur Soziologie des Alltags. Opladen.

Hannappel, Hans/Melenk, Hartmut (1984): Alltagssprache. Semantische Grundbegriffe und Analysebeispiele. 2. überarb. Aufl. München. (UTB. 800). 
Hard, Gerhard (1970): Die "Landschaft" der Sprache und die "Landschaft" der Geographen. Semantische und forschungslogische Studien zu einigen zentralen Denkfiguren in der deutschen geographischen Literatur. Bonn. (Colloquium Geographicum. 11).

Hartmann, Dietrich (1980): Über den Einfluß von Fachsprachen auf die Gemeinsprache. Semantische und variationstheoretische Überlegungen zu einem wenig erforschten Zusammenhang. In: Gnutzmann, Claus/Turner, John (Hrsg.): Fachsprachen und ihre Anwendung. Tübingen. (TBL. 144), S. 27-48.

Heller, Klaus (1970): Der Wortschatz unter dem Aspekt des Fachwortes - Versuch einer Systematik. In: Wissenschaftliche Zeitschrift der Karl-MarxUniversität Leipzig, Gesellschafts- und Sprachwissenschaftliche Reihe, 19, S. 531-544. - Auch in: von Hahn (1981), S. 218-238.

Henne, Helmut/Mentrup, Wolfgang/Möhn, Dieter/Weinrich, Harald (Hrsg.) (1978): Interdisziplinäres deutsches Wörterbuch in der Diskussion. Düsseldorf. (Sprache der Gegenwart. 45).

Hess-Lüttich, Ernest W.B. (1987): Angewandte Sprachsoziologie. Eine Einführung in linguistische, soziologische und pädagogische Ansätze. Stuttgart.

Hoffmann, Lothar (1985): Kommunikationsmittel Fachsprache. Eine Einführung. 2. völlig neu bearb. Aufl. Tübingen. (Forum für Fachsprachen-Forschung. 1). (1. Auf. Berlin 1976).

Hoffmann, Lothar (1988): Vom Fachwort zum Fachtext. Beiträge zur Angewandten Linguistik. Tübingen. (Forum für Fachsprachen-For8chung. 5).

Hoffmann, Lothar (1988 [1977] ): Leipziger Thesen zur fachsprachlichen Forschung. [1977] . In: Hoffmann (1988), S. 19-22.

Hoffmann, Lothar (1988 [1982] ): Probleme und Methoden der FachsprachenForschung. [1982] . In: Hoffmann (1988), S. 23-34.

Hoffmann, Lothar (1988 [1984] ): Vom Fachtext zur Fachtextsorte. [1984] . In: Hoffmann (1988), S. 131-144.

Hoffmann, Lothar (1988 [1985] ): Der Platz der Fachsprachen-Forschung in der Angewandten Sprachwissenschaft. [1985] . In: Hoffmann (1988), S. 35-48.

Ickler, Theodor (1984): Deutsch als Fremdsprache. Eine Einführung in das Studium. Tübingen. (Germanistische Arbeitshefte. 29). 
Ischreyt, Heinz (1965): Studien zum Verhältnis von Sprache und Technik. Institutionelle Sprachlenkung in der Terminologie der Technik. Düsseldorf. (Sprache und Gemeinschaft. Studien. IV).

Jakobson, Roman (1974): Zur Notwendigkeit einer sachlichen und terminologischen Unterscheidung. In: Jakobson, Roman: Aufsätze zur Linguistik und Poetik. Hrsg. u. eingel. v. Wolfgang Raible. München. (Sammlung Dialog. 71), S. 279-280.

Kalverkämper, Hartwig (1978): Die Problematik von Fachsprache und Gemeinsprache. In: Sprachwissenschaft 3, S. 406-444.

Kalverkämper, Hartwig (1979): Der Begriff der Fachlichkeit in der Fachsprachen-Linguistik - Tradition, Kritik und Methoden-Ausblick. In: Fachsprache. Sonderheft 1: „Zweites Europäisches Fachsprachensymposium, Bielefeld 24.-26.9. 1979". Wien, S. 51-71.

Kalverkämper, Hartwig (1980a): Die Axiomatik der Fachsprachen-Forschung. In: Fachsprache 2, S. 1-20.

Kalverkämper, Hartwig (1980b): Das Wortfeld der Fachlichkeit im Französischen. Ein Beitrag der Wortfeldforschung zur Methodologie der Fachsprachenlinguistik. In: Sprachwissenschaft 5, S. 415-496.

Kalverkämper, Hartwig (1982): Fachsprachen und Textsorten. In: Høedt, Jørgen/Lundquist, Lita/Picht, Heribert/Qvistgaard, Jacques (Eds.): Proceedings of the 3rd European Symposium on LSP, Copenhagen, August 1981: „Pragmatics and LSP". Copenhagen, S. 105-168.

Kalverkämper, Hartwig (1983): Textuelle Fachsprachen-Linguistik als Aufgabe. In: Schlieben-Lange, Brigitte/Kreuzer, Helmut (Hrsg.): Fachsprache und Fachliteratur. Göttingen. (Zeitschrift für Literaturwissenschaft und Linguistik 13, Heft 51/52), S. 124-166.

Kalverkämper, Hartwig (1987): Vom Terminus zum Text. In: Sprissler, Manfred (Hrsg.): Standpunkte der Fachsprachenforschung. Tübingen. (Forum Angewandte Linguistik. 11), S. 39-78.

Kalverkämper, Hartwig (1988a): Fachexterne Kommunikation als Maßstab einer Fachsprachen-Hermeneutik. Verständlichkeit kernphysikalischer Fakten in spanischen Zeitungstexten. In: Kalverkämper, Hartwig (Hrsg.): Fachsprachen in der Romania. Tübingen. (Forum für Fachsprachen-Forschung. 8), S. 151-193.

Kalverkämper, Hartwig (1988b): Verständlichkeit, Verständnis und Verständigung im Fadenkreuz: der Wissenschaftstransfer. In: Kodikas/Code, Ars Semeiotica. An International Journal of Semiotics 11, S. 311-325. 
Kalverkämper, Hartwig (1989a): Merkmale eines europäischen 'Fach'-Begriffs. In: Fachsprache - Fremdsprache - Muttersprache. (Schriftenreihe der Sektion Angewandte Sprachwissenschaft und des Instituts für Deutsche Fachsprache an der Technischen Universität Dresden). Heft 9/10: VII. Internationale Konferenz "Angewandte Sprachwissenschaft und fachsprachliche Ausbildung" an der Sektion Angewandte Sprachwissenschaft der Technischen Universität Dresden, 6.-8. September 1988, Teil 1 (Plenarvorträge). Dresden, S. 74-94.

Kalverkämper, Hartwig (1989b): Diatechnische Markierungen im allgemeinen Wörterbuch. In: Hausmann, Franz Josef/Reichmann, Oskar/Wiegand, Herbert Ernst/Zgusta, Ladislav (Hrsg.): Wörterbücher - Dictionaries Dictionnaires. Ein internationales Handbuch zur Lexikographie. BerlinNew York. (HSK - Handbücher zur Sprach- und Kommunikationswissenschaft. 5,1). Artikel 58, S. 680-688.

Kalverkämper, Hartwig (1990): Der Begriff der 'Fachlichkeit' im modernen Italienischen. Lexikalische Systematik und textuelle Integration. In: Quaderni di Semantica. Rivista internazionale di semantica teorica e applicata 11, S. 83-119.

Kalverkämper, Hartwig/Weinrich, Harald (Hrsg.) (1986): Deutsch als Wissenschaftssprache. 25. Konstanzer Literaturgespräch des Buchhandels, 1985. Tübingen. (Forum für Fachsprachen-Forschung. 3).

Klappenbach, Ruth/Steinitz, Wolfgang (Hrsg.) (1976): Wörterbuch der deutschen Gegenwartssprache. Band 5. Berlin.

Laermann, Klaus (1975): Alltags-Zeit. Bemerkungen über die unauffälligste Form sozialen Zwangs. In: Kursbuch 41, S. 87-105.

Mann, Gunter (1982): Dilettant und Wissenschaft. In: Ziegler, Willi (Hrsg.): Biologie für den Menschen. Frankfurt am Main. (Aufsätze und Reden der Senckenbergischen Naturforschenden Gesellschaft. 31), S. 49-72.

Mentrup, Wolfgang (1978a): Überlegungen zur lexikographischen Erfassung der Gemeinsprache und der Fachsprachen. In: Henne/Mentrup/Möhn/ Weinrich (1978), S. 48-77.

Mentrup, Wolfgang (1978b): Korpora und Belegsammlungen. In: Henne/ Mentrup/Möhn/Weinrich (1978), S. 195-232.

Mentrup, Wolfgang (Hrsg.) (1979): Fachsprachen und Gemeinsprache. Jahrbuch 1978 des Instituts für deutsche Sprache [Mannheim] . Düsseldorf. (Sprache der Gegenwart. 46).

Möhn, Dieter (1981[1968] ): Fach- und Gemeinsprache. Zur Emanzipation und Isolation der Sprache. [1968] . In: von Hahn (1981), S. 172-217. 
Möhn, Dieter/Pelka, Roland (1984): Fachsprachen. Eine Einführung. Tübingen. (Germanistische Arbeitshefte. 30).

Paul, Hermann (1880): Prinzipien der Sprachgeschichte. Freiburg. (9. unveränd. Aut. Tübingen 1975).

Picht, Robert (1989): Kultur- und Landeswissenschaften. In: Bausch, KarlRichard/Christ, Herbert/Hüllen, Werner/Krumm, Hans-Jürgen (Hrsg.): Handbuch Fremdsprachenunterricht. Tübingen. (UTB. Große Reihe). Kap. 9, S. 54-60.

von Polenz, Peter (1981): Über die Jargonisierung von Wissenschaftssprache und wider die Deagentivierung. In: Bungarten, Theo (Hrsg.): Wissenschaftssprache. Beiträge zur Methodologie, theoretischen Fundierung und Deskription. München, S. 85-110.

Quintilianus, Marcus Fabius [1972-1975] : Institutionis oratoriae libri XII. Ausbildung des Redners. Zwölf Bücher. Hrsg. u. übers. v. Helmut Rahn. I: Buch I-VI (1972); II: Buch VII-XII (1975). Darmstadt. (Texte zur Forschung. 2, 3).

Raible, Wolfgang (1980): Was sind Gattungen? Eine Antwort aus semiotischer und textlinguistischer Sicht. In: Poetica 12, S. 320-349.

Raible, Wolfgang (1981): Von der Allgegenwart des Gegensinns (und einiger anderer Relationen). Strategien zur Einordnung semantischer Information. In: Zeitschrift für romanische Philologie 97, S. 1-40.

Reinhardt, Werner (1966): Produktive verbale Wortbildungstypen in der Fachsprache der Technik. In: Wissenschaftliche Zeitschrift der Pädagogischen Hochschule Potsdam, Gesellschafts- und Sprachwissenschaftliche Reihe 10, Heft 2, S. 183-195.

Ritz, Hans (1986): Die Geschichte vom Rotkäppchen. Ursprünge, Analysen, Parodien eines Märchens. 2. Aufl. München. (Heyne Allgemeine Reihe. 6603).

Seibicke, Wilfried (1959): Fachsprache und Gemeinsprache. In: Muttersprache, 70-84. - Auch in: von Hahn (1981), S. 40-66.

Steger, Hugo (1988): Erscheinungsformen der deutschen Sprache. 'Alltagssprache' - 'Fachsprache' - 'Standardsprache' - 'Dialekt' und andere Gliederungstermini. In: Deutsche Sprache 16, S. 289-319.

Toellner, Richard (1986): Liebhaber und Wissenschaft. Zur Rolle des Amateurs in der Geschichte der Wissenschaften. In: Berichte zur Wissenschaftsgeschichte 9, S. 137-145. 
Trier, Jost (1951): Lehm. Etymologien zum Fachwerk. Marburg. (Münstersche Forschungen. 3).

Trier, Jost (1981): Wege der Etymologie. Nach der hinterlassenen Druckvorlage mit einem Nachwort hrsg. v. Hans Schwarz. Berlin. (Philologische Studien und Quellen. 101).

Weingarten, Elmar/Sack, Fritz/Schenkein, Jim (Hrsg.) (1976): Ethnomethodologie. Beiträge zu einer Soziologie des Alltagshandelns. Frankfurt. (Suhrkamp Taschenbücher Wissenschaft. 71).

Weinrich, Harald (1976a): Sprache in Texten. Stuttgart.

Weinrich, Harald (1976b): Die Wahrheit der Wörterbücher. In: Probleme der Lexikologie und Lexikographie. Jahrbuch 1975 des Instituts für deutsche Sprache [Mannheim] . Düsseldorf. (Sprache der Gegenwart. 39), S. 347-371.

Weinrich, Harald (1976c): Um einen linguistischen Handlungsbegriff. In: Weinrich (1976a), S. 21-44.

Weinrich, Harald (1976d): Von der Alltäglichkeit der Metasprache. In: Weinrich (1976a), S. 90-112.

Weinrich, Harald (1978): Plädoyer für ein interdisziplinäres Wörterbuch der deutschen Sprache. In: Henne/Mentrup/Möhn/Weinrich (1978), S. 1130 .

Weinrich, Harald (1985): Wege der Sprachkultur. Stuttgart.

Weinrich, Harald (1985 [1981] ): Fremdsprachen für den Alltag und der Alltag des Fremdsprachenunterrichts. [1981] . In: Weinrich (1985), S. 265-289.

Wiegand, Herbert Ernst (1988): Was eigentlich ist Fachlexikographie? Mit Hinweisen zum Verhältnis von sprachlichem und enzyklopädischem Wissen. In: Munske, Horst Haider/von Polenz, Peter/Reichmann, Oskar/Hildebrandt, Reiner (Hrsg.): Deutscher Wortschatz. Lexikologische Studien. Ludwig Erich Schmitt zum 80. Geburtstag von seinen Marburger Schülern. Berlin-New York, S. 729-790.

Wierlacher, Alois (1980): Ästhetik des Alltags - Ansichten eines vernachlässigten Konzepts. In: Jahrbuch Deutsch als Fremdsprache 6, S. 1-7.

Wimmer, Rainer (1979): Das Verhältnis von Fachsprache und Gemeinsprache in Lehrtexten. In: Mentrup (1979), S. 246-275. 
Wolf, Lothar (1979): Fachvokabular und Sprachgemeinschaft. In: Höłler, Manfred/Vernay, Henri/Wolf, Lothar (Hrsg.): Festschrift Kurt Baldinger zum 60. Geburtstag, 17. November 1979. Band II. Tübingen, S. 917933.

Wüster, Eugen (1973): Kaleidoskop der Fachsprachen. In: Drozd/Seibicke (1973), S. VIII-X. 